\title{
A Novel Feature-Based Detector for Underwater Acoustic Beacon Signals Using Superimposed Envelope Spectrum of Multi-Pulses
}

\author{
Shuai Yao * and Yinjia Liu
}

check for updates

Citation: Yao, S.; Liu, Y. A Novel

Feature-Based Detector for

Underwater Acoustic Beacon Signals Using Superimposed Envelope Spectrum of Multi-Pulses. J. Mar. Sci. Eng. 2021, 9, 1337. https://doi.org/ 10.3390/jmse9121337

Academic Editor: Philippe Blondel

Received: 1 November 2021

Accepted: 26 November 2021

Published: 28 November 2021

Publisher's Note: MDPI stays neutral with regard to jurisdictional claims in published maps and institutional affiliations.

Copyright: (c) 2021 by the authors. Licensee MDPI, Basel, Switzerland. This article is an open access article distributed under the terms and conditions of the Creative Commons Attribution (CC BY) license (https:/ / creativecommons.org/licenses/by/ $4.0 /)$.
Key Laboratory of Underwater Acoustic Signal Processing of Ministry of Education, Southeast University, Nanjing 210096, China; 13171883@seu.edu.cn

* Correspondence: 101012104@seu.edu.cn

\begin{abstract}
For tackling the challenge of in-time searching a sea-crashed plane, it is critical to develop a convenient and reliable detector for the underwater beacon signal. In the application of signal detection, a conventional detector such as linear correlation (LC) is used based on the assumption of Gaussian white noise, but it has turned out to be a poor choice in a sophisticated underwater environment. To address this issue, a novel feature-based detector using superimposed envelope spectrum (SES) of multi-pulses is proposed in this paper. The proposed detector firstly extracts the envelopes of the received multi-pulse signals and superimposes the envelopes according to the known period. Then, the harmonic features of the SES are derived and utilized in the feature judgment to make the final decision. The proposed method is evaluated together with several existing state-of-the-art detectors, including the matched filter (MF), the generalized likelihood ratio test (GRLT) detector, and the periodogram of the directly dislocation superposition (PDDS) detectors with constant false alarm probability. Compared with the conventional detectors, it is found that the proposed SES detector is more robust against the colored noise, the random phase, and the channel distortions caused by the sophisticated underwater environment. Simulation results show that, given a detection probability value of $90 \%$ and a false alarm probability value of $1 \%$, the proposed detector shows a gain of 3-12 dB compared with the best one of the MF, GRLT, and the PDDS detectors under distorted channels in terms of signal-to-noise ratio (SNR) requirements, respectively. Experimental results based on lake trial data have also verified the validity and feasibility of the proposed feature-based detector.
\end{abstract}

Keywords: beacon signal detection; superimposed envelope spectrum (SES); ocean colored noise; ocean channel distortion; line spectrum

\section{Introduction}

The beacon signal has been widely applied in many engineering applications. For example, when a plane falls into the water with an explosion or collision, the underwater acoustic beacon in the black box will activate automatically and emit a specific highfrequency pulse signal constantly until the battery runs out. In many rescue operations, the fast and effective detection of this specific beacon signal is required [1-3]. However, the detection of the beacon signal is restricted by the short working time and limited working distance, and the long-distance propagation and complicated marine environment make the detection more difficult [4]. From this perspective, establishing a convenient and reliable detector for the beacon signal is critical.

The detection of a specific high-frequency underwater acoustic beacon signal can be modelled as the problem of detecting a deterministic signal with unknown amplitude and random initial phase in noise. It is a common and fundamental problem in many fields, such as radar, sonar, communications, and image processing [5,6]. In radar and sonar fields, it is a problem of binary hypothesis testing. There are two major approaches to 
binary hypothesis testing. The first one is termed as the Bayesian approach. It considers the unknown parameters as realizations of random variables and assigns a prior PDF. The second one is termed as the generalized likelihood ratio test (GLRT). The GLRT estimates the unknown parameters for use in a likelihood ratio test [5]. The Bayesian approach requires prior knowledge of the unknown parameters, whereas the GLRT does not. Therefore, the GLRT has been more widely used due to its ease of implementation and less restrictive assumptions [5,7]. Based on a white Gaussian noise assumption, the GLRT reduces to a matched filter $(\mathrm{MF})$ receiver or a replica correlator $(\mathrm{RC})$ compared against a threshold [8-10]. For applications in which the signal is under the designer's control (i.e., the known signal assumption is valid), the MF has found extensive use. Nevertheless, as for underwater acoustic noise (UWAN), studies show that they perform impulsive and colored characteristics when polluted by humanmade noise or natural ambient scenarios [11-13]. In this case, techniques optimized for the additive white Gaussian noise (AWGN) model will be suboptimal and the detection performance decreases significantly when the noise is colored or impulsive [14]. Therefore, it is worth exploring detection methods with outstanding robustness to impulsive and colored noise.

With respect to non-Gaussian noise in the signal, many studies are carried out to improve the detection performance. Improvements on the conventional linear correlation (LC) detector yield nonlinear detectors, such as locally optimal (LO) detector and sign correlator (SC) detector. In the presence of non-Gaussian noise, it is proven that the LO detector achieves a better performance than the LC detector [15]. In [16], Al-Aboosi et al. attempted to build a Gaussian noise injection detector (GNID) based on noise-enhanced signal detection. The noise-enhanced signal detection is carried out by using a pre-whitening filter, a time-frequency denoising method based on S-transform, and an inverse whitening filter. It was found that the GNID showed a better performance than the LO, SC, and LC detectors for detecting a time-varying signal. Recently, studies have considered both colored and non-Gaussian properties and focused on optimal detectors without using the noise probability density function (PDF) information. In [17], a threshold-system-based detector (TD) was proposed for detecting a known deterministic signal in independent non-Gaussian noise. The proposed TD was proven to be robust against non-Gaussian noise. It was shown that, even without knowing the noise pdf, the proposed TD shows close performance to the optimal detector designed with the known pdf information [17].

There are many interferential factors in addition to noise. In the underwater channel, two kinds of distortion mechanisms in the underwater propagation path have been researched $[18,19]$, named fast-fading distortion (FFD) and time-spreading distortion (TSD). FFD is caused by multiple propagation while TSD is caused by the dynamics of the ocean environment. Unlike the noise, the impact of channel distortion on the beacon signal is not additive. To address the channel distortions problem, Baggenstoss [18] and Friedlander [19] proposed and examined two detectors, respectively termed as segmented replica correlator (SRC) and replica correlation integration (RCI) detector. It had been proved that the SRC detector performs better in a FFD channel while the RCI detector outperforms the SRC detector in a TSD channel. However, both SRC and RCI are the coherent matched filter, and they would show a performance degradation for dealing with the distorted underwater acoustic beacon signals (sinusoidal). Besides, relative motion between the transmitter and receiver brings about the Doppler effect, leading to undesirable frequency shifting and additional frequency spreading [12]. The Doppler effect is unavoidable because there is relative motion caused by waves, currents, and tides along the spreading path [12]. With no information about the target speed, many reference signals are needed to correlate with the received signal, which results in an increased computational burden. To further improve the performance of detection, both the known frequency characteristic and the time characteristic can be used. In [20], based on the known periodicity of the beacon signal, a directly dislocation superposition (DDS) method was proposed to extract the beacon signal accurately. This method significantly enhances the concerned signal, but it demands 
strict alignment in the superimposition process for a good process gain. However, it is very difficult to achieve strict alignment due to the random phase or the channel distortions.

In conclusion, the conventional detectors are sensitive to the colored noise, the random phase, or the channel distortions. To address this issue, considering the fact that the underwater acoustic beacon signals are emitted constantly once the black box is activated until the battery runs out, a novel feature-based detector is proposed by using the superimposed envelope spectrum (SES) of multi-pulse signals. The proposed detector involves firstly extracting the envelopes of the received signal by using an improved Hilbert transform [21] and superimposing the envelopes according to the same time interval. Then, the line spectrum is detected based on the theoretical analysis of the superimposed envelope spectrum (SES) features. Eventually, a feature judgment based on the line spectrum is used to make the final decision for the received time series. Both computer simulations and experiments based on lake trial data are presented to verify its performance. It is found that the proposed detector shows a more stable performance in colored noise and distorted channels than the existing state-of-the-art methods, including the MF detector, the GRLT detector with constant false alarm probability, and the DDS method proposed in [20]. Simulation results show that, given a detection probability value of $90 \%$ and a false alarm probability value of $1 \%$, the proposed detector shows a gain of 3-12 $\mathrm{dB}$ compared with the best one of the MF, GRLT, and the DDS detectors under distorted channels in terms of signal-to-noise ratio (SNR) requirements, respectively.

The rest of the paper is organized as follows: in Section 2, received signal models are presented and analyzed in a channel with AWGN, a channel with colored noise, and distorted channels, respectively. Section 2 also describes the principles and characteristics of two conventional signal detection methods, including the MF and the GLRT methods. Detailed designs and implementations of the proposed feature-based detector are presented in Section 3. Simulations and experiments based on real lake trial data were conducted to validate the effectiveness and performance of the proposed method in Section 4. Finally, Section 5 concludes the paper.

\section{Signal Model and Algorithm Fundamentals}

In this section, we derive the models of the received underwater acoustic beacon signal under different channels, and two conventional detectors, including the MF and GLRT, are presented, which are widely used in the detection of the deterministic signal.

\subsection{Underwater Acoustic Beacon Signal}

Propagated through the ideal underwater channel, the model for the received multipulse underwater acoustic beacon signal is given as

$$
s(t)=\sum_{n=0}^{N-1} s_{n}(t)=\sum_{n=0}^{N-1} A_{n} \mathrm{e}^{\mathrm{j}\left(2 \pi f_{0} t+\varphi_{n}\right)} \operatorname{rect}\left(\frac{t-\tau / 2}{\tau}-\frac{n T}{\tau}\right),
$$

where $N$ denotes the number of the received pulses, and where $s_{n}(t)$ is the $n$th sub-pulse defined by

$$
s_{n}(t)=A_{n} \mathrm{e}^{\mathrm{j}\left(2 \pi f_{0} t+\varphi_{n}\right)} \operatorname{rect}\left(\frac{t-\tau / 2}{\tau}-\frac{n T}{\tau}\right),
$$

where $\operatorname{rect}(t / \tau)$ is the rectangular window function of width $\tau, A_{n}$ and $\varphi_{n}$ are, respectively, the magnitude and initial phase of the $n$th sub-pulse, $\tau$ and $T$ are, respectively, the pulse duration and the pulse period, and $f_{0}$ is the frequency. In the ideal channel, $A_{n}$ and $\varphi_{n}$ are unknown, but $A_{n}$ is identical for each sub-pulse while the difference between two adjacent $\varphi_{n}$ are identical. The known values of the parameters $f_{0}, \tau$, and $T$ of the underwater acoustic beacon are, respectively, $37.5 \mathrm{kHz}, 10 \mathrm{~ms}$, and $1 \mathrm{~s}$ [20]. The ideal model of multi-pulse beacon signal is shown in Figure 1. 


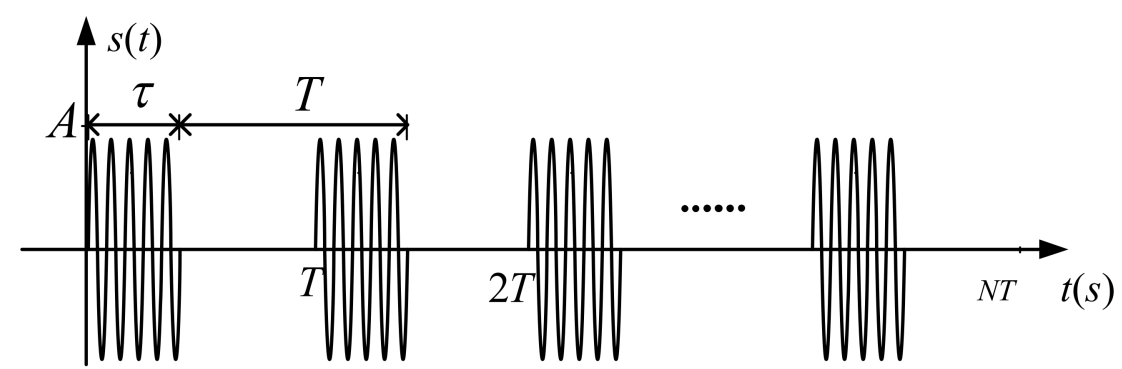

Figure 1. The ideal model of the multi-pulse beacon signal.

Additionally, the received underwater acoustic beacon signals usually suffer from the Doppler effect caused by the relative motions. The received underwater beacon signals with Doppler effect is expressed as

$$
s_{d}(t)=s(b t) \text {, }
$$

with $b$ being the stretch factor of signal time scale defined by $b=1+2 v / c$, where $v$ is the relative movement speed between the beacon searcher and the beacon, and $c$ is the speed of sound in the water. In this paper, we use $s_{d}(t)$ to represent the received underwater acoustic beacon signals with Doppler effect.

\subsection{Noise and Distortions}

Let $x(t)$ denote the received signal under the uncertainty of signal presence. The detection problem could be summarized as a binary hypothesis. In addition, the colored noise and channel distortions should be taken into account for detecting the underwater acoustic beacon signals. Here, three cases of undesired influences on the received underwater beacon signals are presented. Both colored noise and channel distortions are considered, which is critical to proceed with the detection system and simulations.

Case 1: Additive white Gaussian noise (AWGN)

The first case is the ideal one, where the noise is the AWGN, and none of the channel distortions are considered. The received underwater beacon signal with only AWGN is represented as

$$
x(t)=s_{d}(t)+w(t),
$$

where $w(t)$ is the AWGN, and it is assumed to be zero-mean and with variance $\sigma^{2}$.

Case 2: Colored noise

As described in the introduction section, noise accompanying the beacon signal is always non-Gaussian and nonwhite. Hence, the case of colored noise is the concern in this paper. The received underwater beacon signal with colored noise is given by

$$
x(t)=s_{d}(t)+w_{c}(t),
$$

where $w_{c}(t)$ denotes the colored noise. The spectrum $P_{c}(f)$ of the colored marine noise $w_{\mathcal{c}}(t)$ can be described as the Esc model with three parameters [22,23], that is,

$$
P_{c}(f)=\lambda\left(\frac{f_{m}+\kappa\left(f+f_{c}\right)}{f_{m}^{2}+\left(f+f_{c}\right)^{2}}+\frac{f_{m}-\kappa\left(f-f_{c}\right)}{f_{m}^{2}+\left(f-f_{c}\right)^{2}}\right),
$$

where $f_{m}, f_{c}$, and $\kappa$ are three parameters in this model, which determine the shape and size of $P_{c}(f)$, and $\lambda$ denotes the spectra level. More concretely, $f_{c}$ determines the peak position of $P_{c}(f), f_{m}$ determines the level of sharpness and height of spectral peak. For the same $f_{c}$, the larger the $f_{m}$ is, the lower and smoother the spectral peak is, and on the contrary, the smaller the $f_{m}$ is, the higher and steeper the spectral peak is. $\kappa$ determines the relative ratio of high- and low-power spectrum amplitudes. For the same $f_{m}$ and $f_{c}$, if $\kappa>0$, the low-frequency end of the curve is raised and the high-frequency end is fallen; if $\kappa<0$, the 
result is the opposite. The Esc model is commonly applied to simulate the colored marine noise and the colored ship-radiated noise [24-26].

The normalized spectrum of the received beacon signal $(37.5 \mathrm{kHz})$ with the colored noise is shown in Figure 2. The colored noise is obtained based on the aforementioned Esc model, with the three parameters, respectively, set as $f_{m}=300 \mathrm{~Hz}, f_{c}=500 \mathrm{~Hz}$, and $\kappa<0$; and the spectrum level $\lambda$ is set to 1 . The sample frequency $f_{s}$ is set to $128 \mathrm{kHz}$. Note that $x(t)$ contains the desired signal at $37.5 \mathrm{kHz}$, but the power spectral density decays with frequency, and the signal energy is overwhelmed by noise. Therefore, it is difficult to detect the signal directly through spectrum analysis and peak detection for the line spectrum of the signal.

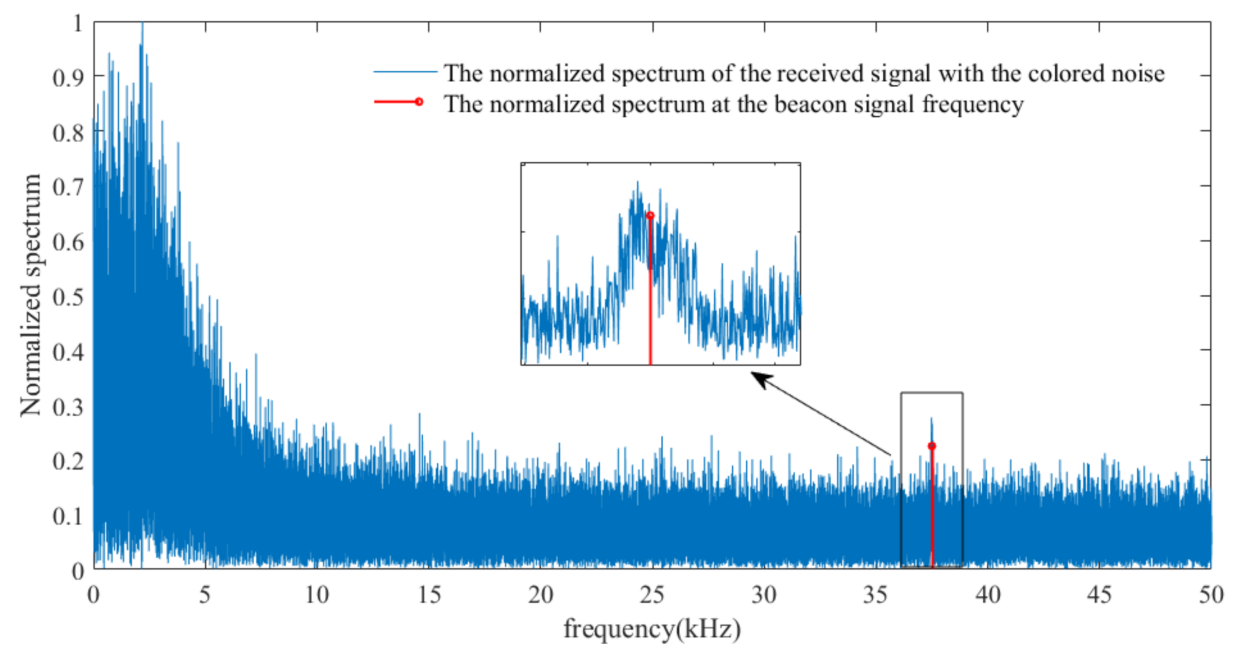

Figure 2. The normalized spectrum of the received signal with the colored noise.

Case 3: Channel distortions

The received underwater beacon signals undergo distortions during the underwater channel propagation and reflection, and we refer to two common channel distortion models as FFD and TSD [18]. In this paper, FFD and TSD channels are considered, respectively.

FFD is caused by dynamics of the underwater environment or uncompensated dynamics of the transmitting or receiving platforms. Unlike the noise, the impact of channel distortion on the beacon signal is not additive. In [18], the distorted received signal propagating through the FFD channel can be represented by the modulation of the transmitted signal with time-varying function. The received signal $x(t)$ with FFD distortion could be given by

$$
x(t)=\xi(t) s_{d}(t),
$$

where $\xi(t)$ is a time-varying function characterized by its bandwidth $B_{c}=1 / T_{c}$, where $T_{c}$ is the coherence time.

TSD is another type of distortion, which is caused by multiple propagation paths or occurs when a transmitted waveform impinges on an extended reflector [18]. For the TSD, the received signal may be represented by the convolution of the transmitted signal with a time-spreading function $\eta(t)$. For a time-spreading function $\eta(t)$ of length $T_{s}$, the received signal $x(t)$ is expressed as

$$
x(t)=\int_{u=0}^{T_{s}} \eta_{n}(u) s_{d}(t-u) \mathrm{d} u,
$$

It is assumed that $\eta(t)$ is a realization of a random Gaussian process that remains fixed for each sub-pulse [18]. 


\subsection{Conventional Detectors}

As mentioned in Section 2.2, a known signal detection problem is to determine the presence of a signal in the noise, and a binary hypothesis is used to describe the detection problem, as follows:

$$
\left\{\begin{array}{cc}
H_{0}: & x(t)=w_{x}(t) \\
H_{1}: & x(t)=s_{I}(t)+w_{x}(t)
\end{array},\right.
$$

where $s_{I}(t)$ is the beacon signal and $w_{x}(t)$ is the noise. Let us present two conventional detectors as comparisons before we proceed on the derivation of an optimal detection method for the known beacon signal with noise and distortions. They are the MF and GLRT, and their characteristics are clarified later.

\subsubsection{The MF Detector}

The MF is the optimal detector for the known signal in AWGN. Based on the NeymanPearson (NP) criterion, we decide $H_{1}$ if

$$
L(x)=\frac{p\left(x ; H_{1}\right)}{p\left(x ; H_{0}\right)}>\gamma,
$$

where $p\left(x ; H_{1}\right)$ and $p\left(x ; H_{0}\right)$ are PDFs under each hypothesis, $L(x)$ is the test statistic, and the threshold $\gamma$ can be given by a set $P_{f}$ [27]. Specifically, the NP detector can also be implemented in the form of an MF, which is applied commonly. Its impulse response function and output are, respectively, given as [27]

$$
\begin{aligned}
& h(t)=k s\left(t_{0}-t\right), \\
& y(t)=x(t) * h(t),
\end{aligned}
$$

where $x(t)$ is the received signal, $s(t)$ is the known signal, and $t_{0}$ is the time when the output reaches the maximum SNR. If decision is made at the time $t=t_{0}$, the test statistic $L(x)$ can be rewritten as

$$
L(x)=y\left(t_{0}\right)=k \int_{0}^{t_{0}} s(t) x(t) \mathrm{d} t,
$$

and we decide $H_{1}$ when $L(x)>\gamma$.

\subsubsection{The GLRT Detector}

The MF is the optimal detector in AWGN, but it assumes that all the parameters of the signal are known. However, the received underwater beacon signals could be modeled as the deterministic sinusoidal signal with unknown amplitude and phase, that is

$$
\left\{\begin{array}{ll}
H_{0}: & x(t)=w_{x}(t) \\
H_{1}: & x(t)=s_{I}(t)+w_{x}(t)
\end{array},\right.
$$

where $s_{I}(t)=A \cos \left(2 \pi f_{0} t+\varphi\right)$, and $w_{x}(t)$ is the noise. The amplitude $A$ and the phase $\varphi$ are unknown.

In the case of detecting the deterministic signals with unknown parameters, an optimal detector will usually not exist, and the GLRT, a suboptimal detector, could produce good detection performance. The GLRT decides $H_{1}$ if [27]

$$
\frac{p\left(x ; \hat{A}, \hat{\varphi}, H_{1}\right)}{p\left(x ; H_{0}\right)}>\gamma,
$$


where $p\left(x ; \hat{A}, \hat{\varphi}, H_{1}\right)$ and $p\left(x ; H_{0}\right)$ are the PDFs under $H_{1}$ and $H_{0}$, respectively, $\hat{A}, \hat{\varphi}$ are the maximum likelihood estimation (MLE), $f_{0}$ is not near 0 or $1 / 2$, and $\gamma$ is the threshold. Substituting the expressions of the PDFs and MLEs [27] into Equation (15), it reduces to

$$
I\left(f_{0}\right)=\left.\frac{1}{N_{s}}|| \sum_{n=0}^{N_{s}-1} x(n) \mathrm{e}^{-\mathrm{j} 2 \pi f_{0} n}\right|^{2}>\sigma^{2} \ln \gamma,
$$

where $x(n)$ is the discrete sample of $x(t)$ and has a length of $N_{S}$, and $I\left(f_{0}\right)$ denotes the periodogram of the sinusoidal signal [27]. If we define $\gamma^{\prime}=\sigma^{2} \ln \gamma$, a given false alarm rate $P_{f}$ could determine the threshold $\gamma^{\prime}$, as follows:

$$
P_{f}=\exp \left(-\frac{\gamma^{\prime}}{\sigma^{2}}\right)
$$

Both the MF and GLRT detectors can be implemented for the beacon signal detection with the known frequency information. However, as the signal is interfered with by colored noise and channel distortions, the conventional detectors will show performance degradation and are no longer optimal choices. The MF output and the GLRT periodogram of the beacon signal are, respectively, shown in Figures 3 and 4. For the channels with AWGN, the SNR is set to be $-3 \mathrm{~dB}$. For the channels with Doppler shift, the relative movement speed between the beacon searcher and the beacon $v$ is set to $4 \mathrm{~m} / \mathrm{s}$, and the speed of sound in the water $c$ is set to be $1500 \mathrm{~m} / \mathrm{s}$. For the channels with colored noise, three parameters of the Esc model are, respectively, set as $f_{m}=300 \mathrm{~Hz}, f_{c}=500 \mathrm{~Hz}$, and $\kappa<0$, and the spectrum level $\lambda$ is set to 1 . For the FFD channel, $T_{\mathcal{c}}$ is set to $1.25 \mathrm{~ms}$ and $M_{c}$ is set to 8. For the TSD channel, the length $T_{s}$ of the time-varying function $\eta(t)$ is set to $3.2 \mathrm{~ms}$. The sample frequency $f_{s}$ is set to $128 \mathrm{kHz}$. It can be seen that the MF output and periodogram perform well in an ideal channel but suffer serious time distortion or amplitude distortion in the presence of the Doppler effect, the colored noise, and channel distortions. In this paper, we address this problem by proposing a novel method, and we focus on the robustness and gain of the detection, which is clarified in the following section.

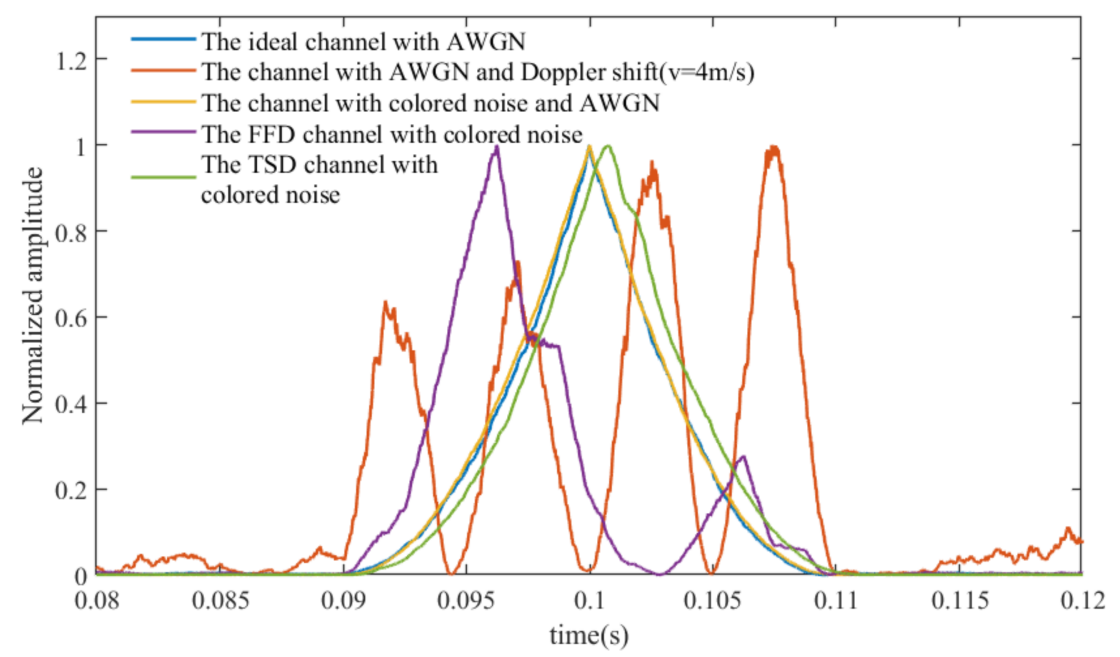

Figure 3. The MF output of beacon signal in various channels. 


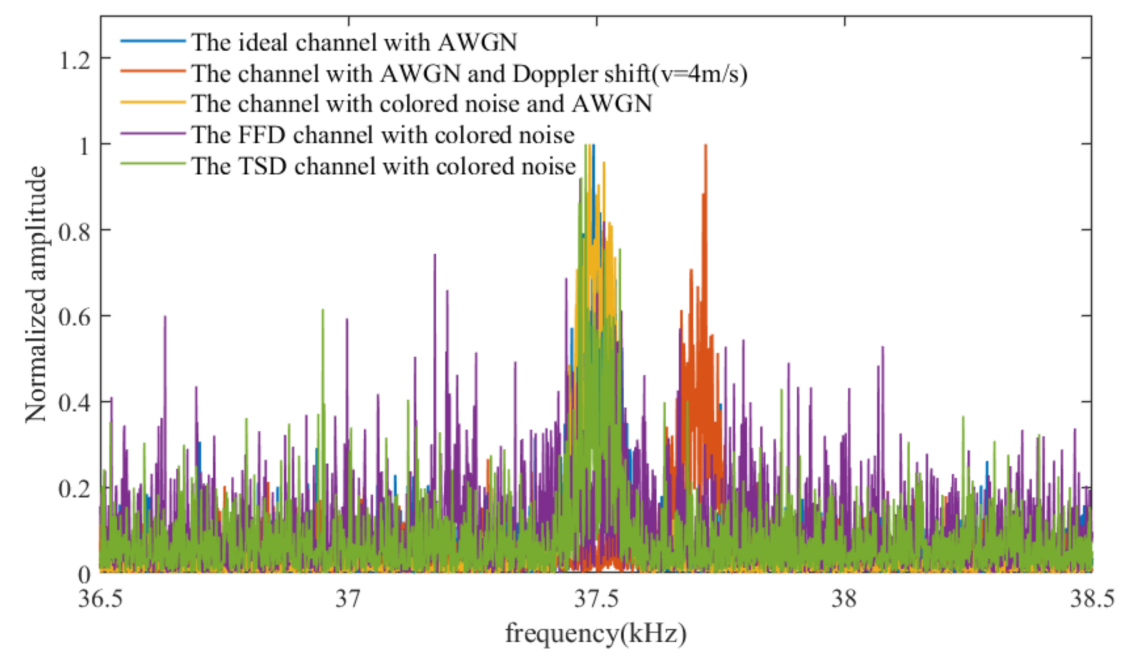

Figure 4. The periodogram of beacon signal in various channels.

\section{The Proposed Detector}

In this section, we propose a feature-based detector based on the superposition method to improve the robustness of the detector against the colored noise and channel distortions. The directly dislocation superposition method has been studied earlier. If there is no random phase, then it suffices to detect the noisy signal with its robust periodicity characteristic. We present here an improved way to retain the superiority of this method. Besides, an analysis of the multi-pulse envelope's feature is also carried out for the proposed method, which is the key to improving detection performance.

\subsection{Beacon Signal Detection Using the Superposition Method}

The periodicity of the multi-pulse beacon signals is robust against noise and channel distortions, which could improve the detection performance and address previous concerns. One effective way to enhance the signal using the periodicity of the multi-pulse beacon signals is the dislocation superposition method. In [20], Zhang applied the directly dislocation superposition (DDS) algorithm to the extraction process. The main idea of this method is the superposition of a series of sub-pulses, and it is given as

$$
Y(t)=\frac{1}{N} \sum_{k=0}^{N-1} x(t+k T), 0<t<T,
$$

where $x(t)$ is the received signal, $T$ denotes the length of superimposed signal, $N$ is the number of superposition times, and $k$ is the number of iterations $(k=0,1,2 \ldots)$. The entire system is simple, with a low computational complexity, and the sinusoidal signal part is enhanced through the superposition. Besides, the signal extraction is proved more accurate compared with traditional methods such as Fourier and wavelet [20]. However, this dislocation superposition algorithm does not take into account the fact that the random phase may exist in the received signal. In the presence of the random phase, the signalenhanced effect will decrease. In addition, if the sub-pulses cannot be aligned perfectly according to the period, the extraction effect will also degrade.

Considering the problems of the superposition of the time waveform directly, we refer to using the envelope of the received signal to superimpose. Accordingly, a novel feature-based detector for the underwater acoustic beacon signal with noise and distortions is proposed. The basic idea of this method is to extract the envelope of the beacon signal and superimpose the envelope instead of the time domain waveform directly, and the spectrum is then analyzed. In the following text, we refer to the superimposed envelope spectrum as SES. With a special envelope extraction method and the properties of SES in the frequency domain, significant detection performance and robustness could be achieved. 


\subsection{The Detector System}

A schematic of the proposed detection system is shown in Figure 5. From Figure 5, it can be seen that the detection system consists of four main parts: envelope extraction, superposition of envelope, feature analysis, and decision-making.

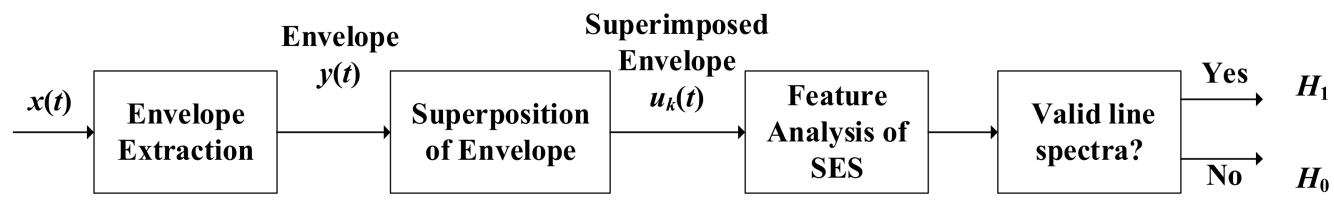

Figure 5. Schematic of the proposed detection system.

As shown in Figure 5, an improved Hilbert transformation (HT) [21] is firstly employed in this detection system to extract the envelope of the multi-pulse beacon signals. A frequency-domain filter is used by the improved HT to extract the envelope. The definition of the frequency-domain filter is given as

$$
H_{F}(j w)=\left\{\begin{array}{cc}
2 & w_{1}<w<w_{2} \\
0 & \text { otherwise }
\end{array},\right.
$$

where $w_{1}$ and $w_{2}$ are the lower and upper limits of the frequency-domain filter. The envelope $y(t)$ of signal can be expressed as

$$
y(t)=\mathcal{I F}\left\{X(j w) H_{F}(j w)\right\},
$$

where $\mathcal{I F}\{\cdot\}$ denotes inverse Fourier transform (IFT), and $X(j w)$ is the complex spectrum of $x(t)$. Then, without loss of generality, we analyze the expression of the envelope $y(t)$ in an ideal situation, that is, the extracted envelope $y(t)$ is approximated as the rectangular pulse train $u(t)$, and it is given as

$$
u(t)=\sum_{n=0}^{N-1} u_{1}(t-n T),
$$

where $u_{1}(t)=\operatorname{Arect}(((t-\tau / 2)) / \tau), 0 \leq t \leq T$. It represents the rectangular envelope of a sub-pulse with pulse duration $\tau$ and pulse period $T$. The extracted envelopes of the multi-pulse beacon signal are shown in Figure 6.

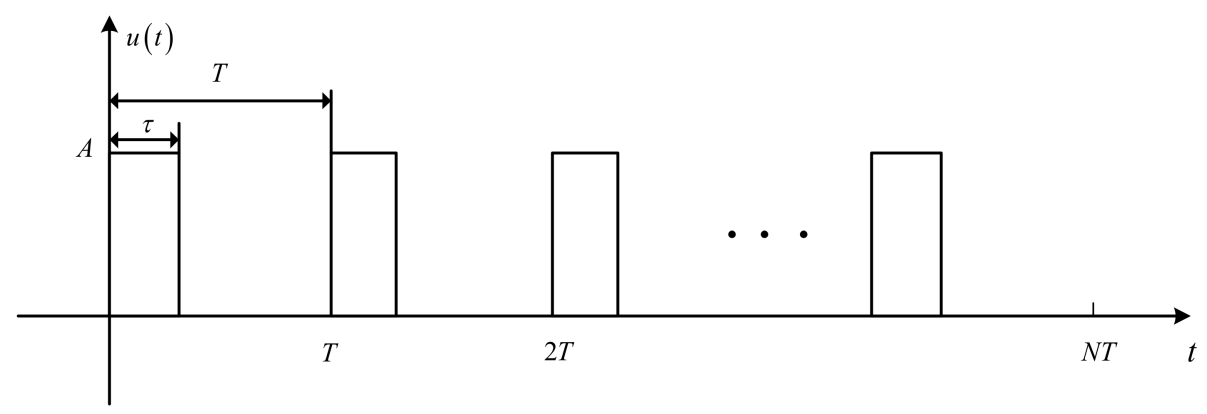

Figure 6. Envelope of the multi-pulse beacon signal $u(t)$.

After the envelope extraction process, the envelope $y(t)$ is approximately a rectangular pulse train $u(t)$ with steady amplitude and period. In this case, the superposition of adjacent sub-pulses will contribute to a high gain of the detection process. Moreover, the spectrum features of the superimposed envelope display a close connection to the periodicity. The analysis below shows that the characteristics of SES can be used to improve the robustness of the detector. 


\subsubsection{Features of SES}

The SES is closely connected to the properties of the beacon signal with periodicity. It is known that periodic signals have some spectrum characteristics in the frequency domain, including the harmonic spectrum. Most importantly, the spectral features of the periodic signals are robust against noise, channel distortions, and misalignment. Here, a detailed analysis on SES is given as follows.

In order to obtain the superposition gain while keeping the periodicity of the signal envelope, the envelopes of multiple pulses are reserved by the superimposition process. If the sub-pulses can be accurately aligned, the superimposition process is shown in Figure 7. As shown in Figure 7, K pulses are reserved after aligned superimposition process, and the superposition result is given as

$$
S_{K}(t)=\sum_{l=1}^{L} U_{l}(t+(l-1) T), 0 \leq t \leq K T,
$$

where

$$
U_{l}(t)=\sum_{k=0}^{K-1} u_{1}(t-k T-(l-1) K T)
$$

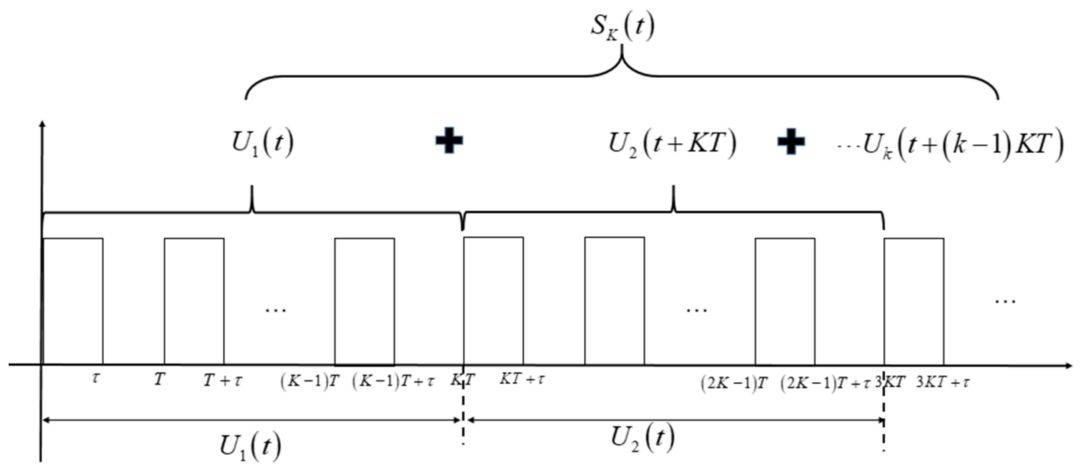

Figure 7. Procedure of aligned superimposition.

$L$ denotes the total number of segments, $L=[N / K]$, and $X$ denotes rounding $X$ to the nearest integers less than or equal to $X$. By inserting $U_{l}(t)$ into Equation (22), we can obtain

$$
S_{K}(t)=L \sum_{k=0}^{K-1} \operatorname{Arect}\left[\frac{(t-\tau / 2-k T)}{\tau}\right],
$$

The $K$-pulse superimposed envelope with aligned superimposition $S_{K}(t)$ is shown in Figure 8.

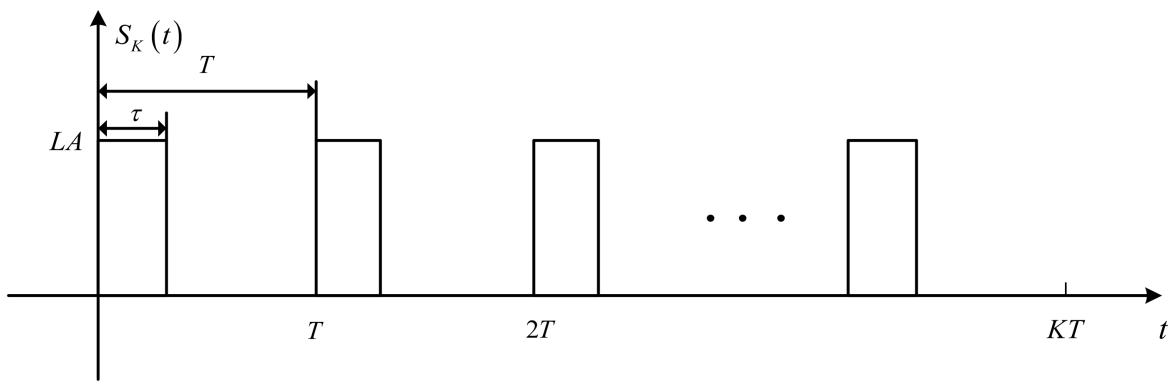

Figure 8. $K$-pulse superimposed envelope $S_{K}(t)$ with aligned superimposition. 
It is difficult to meet accurate alignment during the superimposition process. It is more common that the alignment is not strict or accurate during the superposition process. In this case, the superimposition result $S_{K}^{\prime}(t)$ is given as follows:

$$
\begin{aligned}
S_{K}^{\prime}(t) & =L \sum_{k=0}^{K-1} u_{1}^{\prime}(t-k T) \\
& =L A \sum_{k=0}^{K-1}\left\{\operatorname{rect}\left[\frac{t-\tau_{0} / 2-k T}{\tau_{0}}\right]+\operatorname{rect}\left[\frac{t-T+\left(\tau-\tau_{0}\right) / 2-k T}{\tau-\tau_{0}}\right]\right\},
\end{aligned}
$$

where $u_{1}^{\prime}(t)=u_{1}\left(t-\tau_{0}\right)$ and $\tau_{0}$ denotes the misaligned time. The $K$-pulse superimposed envelope with misaligned superimposition $S_{K}^{\prime}(t)$ is shown in Figure 9.

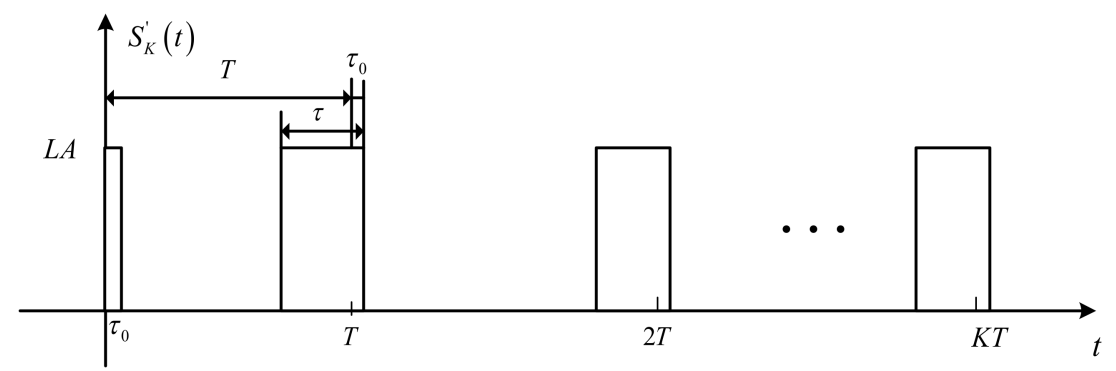

Figure 9. K-pulse superimposed envelope $S_{K}^{\prime}(t)$ with aligned superimposition.

Using the Fourier transform, we can obtain the spectra for $S_{K}(t)$ and $S_{K}^{\prime}(t)$; they are given as follows:

$$
\begin{aligned}
P_{K}(f) & =L U_{1}(f) \sum_{k=0}^{K-1} \mathrm{e}^{-\mathrm{j} 2 \pi f k T} \\
& =L U_{1}(f)\left[\frac{\sin (\pi f T K)}{\sin (\pi f T)}\right] \mathrm{e}^{-\mathrm{j} \pi f[(K-1) T+\tau]}, \\
P_{K}^{\prime}(f) & =L U_{1}^{\prime}(f) \sum_{k=0}^{K-1} \mathrm{e}^{-\mathrm{j} 2 \pi f k T} \\
& =L U_{1}^{\prime}(f)\left[\frac{\sin (\pi f T K)}{\sin (\pi f T)}\right] \mathrm{e}^{-\mathrm{j} \pi f(K-1) T} .
\end{aligned}
$$

The amplitude spectrums are given as

$$
\begin{aligned}
& \left|P_{K}(f)\right|=L\left|U_{1}(f) \frac{\sin (\pi f T K)}{\sin (\pi f T)}\right|, \\
& \left|P_{K}^{\prime}(f)\right|=L\left|U_{1}^{\prime}(f) \frac{\sin (\pi f T K)}{\sin (\pi f T)}\right|,
\end{aligned}
$$

where $U_{1}(f)$ and $U_{1}^{\prime}(f)$ denote the Fourier transform of $u_{1}(t)$ and $u_{1}^{\prime}(t)$, and they are given as

$$
\begin{gathered}
U_{1}(f)=\int_{-\infty}^{+\infty} u_{1}(t) \mathrm{e}^{-\mathrm{j} 2 \pi f t} \mathrm{~d} t, \\
U_{1}^{\prime}(f)=\int_{-\infty}^{+\infty} u_{1}^{\prime}(t) \mathrm{e}^{-\mathrm{j} 2 \pi f t} \mathrm{~d} t=\mathrm{e}^{-\mathrm{j} 2 \pi f \tau_{0}} \int_{-\infty}^{+\infty} u_{1}(t) \mathrm{e}^{-\mathrm{j} 2 \pi f t} \mathrm{~d} t .
\end{gathered}
$$

By substituting Equations (30) and (31) into Equations (28) and (29), respectively, we can obtain

$$
\left|P_{K}^{\prime}(f)\right|=\left|P_{K}(f)\right| .
$$

The amplitude spectrum $\left|P_{K}(f)\right|$ is shown in Figure 10. 


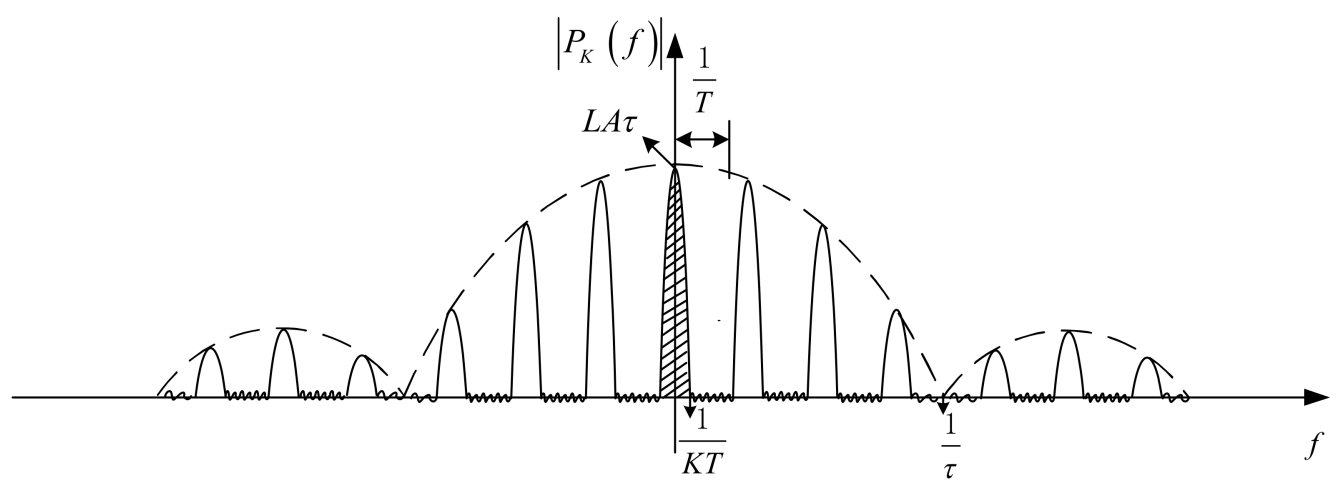

Figure 10. The amplitude spectrum $\left|P_{K}(f)\right|$.

Now, we analyze the properties of SES. As shown by Equation (28) and Figure 10, both $\left|P_{K}(f)\right|$ and $\left|P_{K}^{\prime}(f)\right|$ have a factor $|\sin (\pi f T K) / \sin (\pi f T)|$. Under the modulation of this factor, the SES presents the following properties:

- Comb-shaped: The entire spectrum is in the shape of comb teeth, and each tooth is determined by the corresponding factor $|\sin (\pi f T K) / \sin (\pi f T)|$.

- Harmonic: Each tooth's peak only appears at frequencies that are integer multiple of the fundamental frequency $1 / T$.

- Locally periodic: For the amplitude spectrum within the frequency domain $\left(\frac{n}{\tau}, \frac{n+1}{\tau}\right)$, $n=0,1,2 \ldots$, the interval of any two adjacent teeth is $1 / T$.

- Convergent: The amplitude of each tooth generally decreases with the frequency. When the frequency increases to the infinite, the amplitude of the tooth becomes infinitely smaller.

\subsubsection{Decision-Making}

As mentioned above, the SES of the received signals keeps the harmonic and periodic characteristics. With a complete analysis in the frequency domain, the characteristics of the SES are taken into account for the decision-making part of the detection system. The procedure of decision-making is given as follows:

(1) Set the Frequency Detection Range of the Spectral Lines.

Based on the known parameters $T, \tau$, and $f_{0}$ of the beacon signal, the theoretical SES amplitude is derived in Equation (28). Hence, one can obtain the discrete frequencies where the spectral lines appear, that is $f_{i}=i / T, i=1,2, \ldots$ In this paper, the first three theoretical spectral lines of $f_{i}$ are detected to judge the presence of the beacon signal, which is $f_{i}=i / T,(i=1,2,3)$. Considering the resolution problem, the frequency detection range of each spectral line is $\left[f_{i}-\Delta f, f_{i}+\Delta f\right]$, and the frequency offset $\Delta f$ here is set as $1 /(3 T)$.

(2) Judge the Effectiveness of the Spectral Lines.

To judge the effectiveness of the spectral lines in the set frequency range, the local signal-to-noise ratio (LSNR) is introduced. The LSNR is defined by the ratio of the maximum amplitude of the spectral lines to the average amplitude of other spectral lines in the set frequency range. The LSNR of the searched $i$-th spectral line is expressed as

$$
\operatorname{LSNR}_{i}=\frac{U_{M}(f)}{U_{m}(f)}
$$

where $U_{M}(f)$ denotes the maximum amplitude of the SES in the set frequency range $\left[f_{i}-\Delta f, f_{i}+\Delta f\right]$, and $U_{m}(f)$ denotes the average amplitude of the SES in the range $\left[f_{i}-\Delta f, f_{i}+\Delta f\right]$ with $f \neq f_{i}$. 
If the maximum amplitude value $U_{M}(f)$ is larger than the threshold $\gamma_{U}$, and the $L S N R_{i}$ is larger than the set threshold $\gamma_{L}$, the $i$-th spectral line in set $i$-th frequency range is considered valid. The decision expression is written as follows:

For the $i$-th spectral line at $f_{i}$, if

$$
U_{m}(f)>\gamma_{U}
$$

and

$$
\mathrm{LSNR}_{i}>\gamma_{L},
$$

the spectral line is judged to be valid. Specifically, the values of the thresholds $\gamma_{U}$ and $\gamma_{L}$ are set according to the theoretical SES. The peak amplitude of the theoretical SES is modulated by the function $\sin \mathrm{c}(f \tau)$. Thus, the theoretical amplitude of each peak can be calculated theoretically. Affected by the noise and interferences, the amplitude has some fluctuations but decreases with the frequency. Therefore, the linear decreasing function $(-f \tau+1)$ is used to weight the threshold. The formulas are as follows:

$$
\begin{gathered}
\gamma_{U}=w_{1} \cdot\left|\operatorname{sinc}\left(f_{i} \tau\right)\right|+w_{2} \cdot\left(-\tau f_{i}+1\right), \\
\gamma_{L}=\frac{\gamma_{U}}{U_{m}(f)},
\end{gathered}
$$

where the weights $w_{1}$ and $w_{2}$ are set as 0.5 and 0.5 by Monte Carlo simulations.

If the three detected spectral lines are all judged to be valid, the beacon signal is regarded to be detected, and otherwise it is not in existence.

\section{Simulations and Experiments}

In this section, we evaluate the performance of the proposed detector by comparing it with the existing state-of-the-art detectors, including the MF detector, GLRT detector, and the detector based on periodogram of the directly dislocation superposition (PDDS) for both simulated signals and lake trial data.

The parameters of the beacon signal are, respectively, set as $\tau=10 \mathrm{~ms}, T=1 \mathrm{~s}$, and $f_{0}=37.5 \mathrm{kHz}$. The sample frequency $f_{s}$ is set to be $128 \mathrm{kHz}$. For the channels with Doppler shift, the relative movement speed between the beacon searcher and the beacon $v$ is set to $8 \mathrm{~m} / \mathrm{s}$, and the speed of sound in the water $c$ is set to be $1500 \mathrm{~m} / \mathrm{s}$. For the channels with random phase, the random phase is assumed to be uniformly distributed in $[-\pi, \pi]$.

\subsection{Simulation Results}

Simulations are presented, firstly, to demonstrate the robustness of the proposed detector in various channels, and secondly, to evaluate the performance of the proposed detector by comparing it with other detectors, including the MF detector, the GLRT detector, and the PDDS detector. Channels with AWGN, colored noise, FFD, and TSD are considered, respectively, and various channel models are generated according to Equations (3)-(8). For all of the following given cases, the Doppler shift and the initial phase are taken into account.

The simulation of the AWGN channel is approximated by the Gaussian random process with the specified variance, and the variance $\sigma$ is calculated using the SNR and the signal amplitude $A$. The SNR is defined in decibels $(\mathrm{dB})$ as $S N R=10 \lg \left(A^{2} / 2 \sigma^{2}\right)$. The colored noise is simulated under the Esc model, as given by Equation (6). Three parameters of the Esc model are, respectively, set as $f_{m}=300 \mathrm{~Hz}, f_{c}=500 \mathrm{~Hz}$, and $\kappa<0$, and the spectrum level $\lambda$ is set to 1 . In this paper, Gaussian white noise is used to generate colored noise through the AR model. The power of the generated colored noise is calculated, and then the signal amplitude $A$ is adjusted according to this value and the set SNR.

In this paper, we assume that the time-varying function $\xi(t)$ of the FFD channel is piecewise constant with the length $T_{\mathcal{C}}$, and the number of segments $M_{\mathcal{C}}$ is given by $M_{c}=\tau / T_{\mathcal{c}}$. The piecewise constant is generated by a Gaussian random process with 
zero mean and unit variance. In the simulations, $T_{c}$ is set to $1.25 \mathrm{~ms}$ and $M_{c}$ is set to 8 . The received signal in the FFD channel is simulated by multiplying the signal with the piecewise constant as given by Equation (7). For each pulse of the simulated multi-pulse beacon signals in the FFD channel, different piecewise constants are used. In the simulation of the TSD channel, the length $T_{S}$ of the time-varying function $\eta(t)$ is set to $3.2 \mathrm{~ms}$. For each pulse of the simulated multi-pulse beacon signal in the TSD channel, we also use different random $\eta(t)$ with the same time length $T_{s}$, as given by Equation (8).

Case 1: Envelope and superimposed envelope in various channels.

Firstly, to verify the robustness of the superimposed envelope, the superimposed envelopes in various channels in the time domain are analyzed. The number of processed pulses for detection $N$ is set to 100 and the number of the superimposed sub-pulse $K$ is set to 10 . The SNR is set to $0 \mathrm{~dB}$. The results are shown in Figure 11. Figure 11a presents the superimposition result for the ideal channel with AWGN, Figure $11 \mathrm{~b}$ presents the result for the channel with colored noise and AWGN, Figure 11c corresponds to the FFD channel with colored noise, and Figure 11d corresponds to the TSD channel with colored noise. According to Figure 11, it is easy to see that the superimposed envelopes perform reliably and robustly in all channels. The cases of FFD and TSD channels are slightly worse than the cases for the ideal channel. The envelopes of multi-pulse beacon signal in FFD and TSD channel are distorted seriously. After superimposing, the features of envelopes are enhanced, which can help us to improve the detection performance.

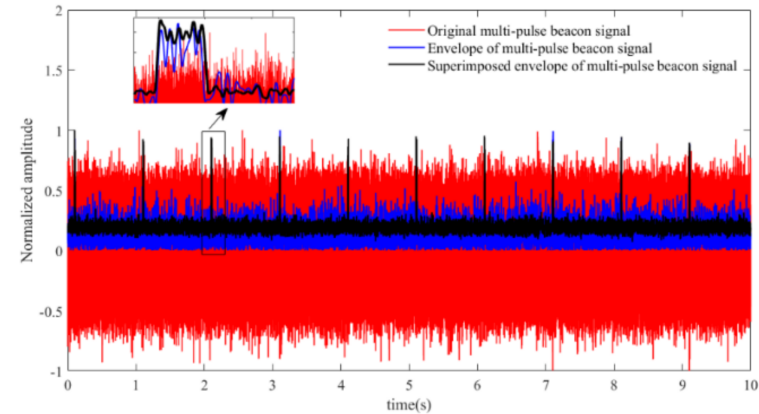

(a)

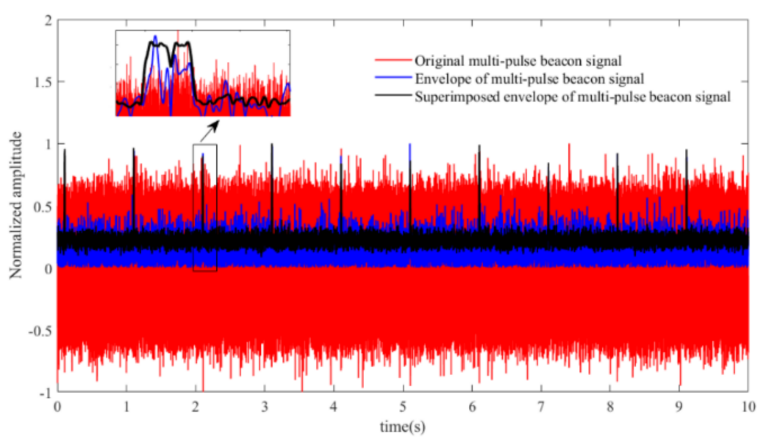

(c)

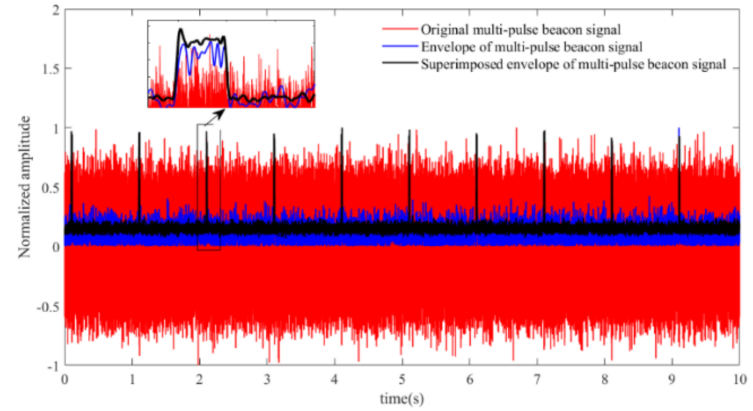

(b)

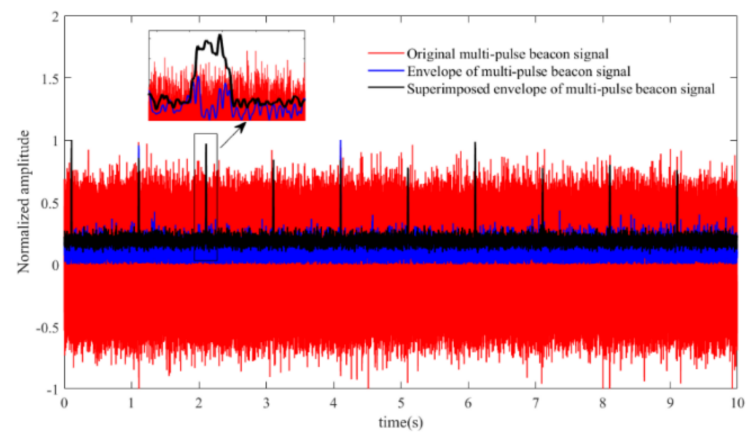

(d)

Figure 11. The superimposed envelopes in various channels: (a) in the ideal channel with AWGN, (b) in the channel with AWGN and colored noise, (c) in the FFD channel with colored noise, and (d) in the TSD channel with colored noise.

Case 2: PDDS and SES in various channels.

As mentioned in Section 3.1, the PDDS detector refers to calculating the periodogram of the directly dislocation superimposed waveforms in the time domain. Therefore, the PDDS directly affects the detection results and the robustness of the detector. The proposed detector relies on the spectrum of the superimposed envelopes. The SES affects the results and robustness of the proposed detector. Thus, we compare the PDDS and the SES results of the multi-pulse signal in various channels. Two cases of the random phase and no random phase are considered, respectively. Simulation results are illustrated in Figures 12-15. 


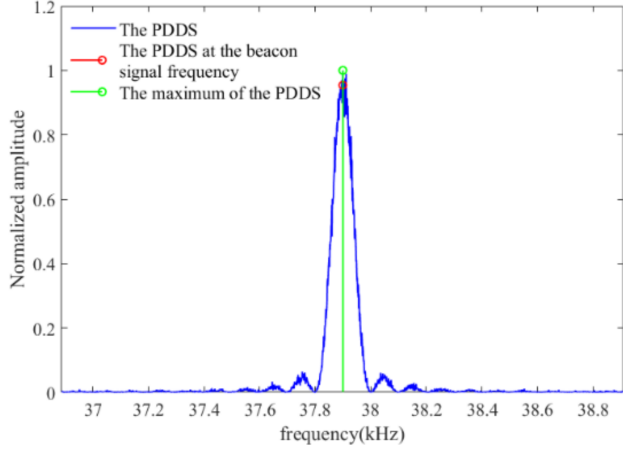

(a)

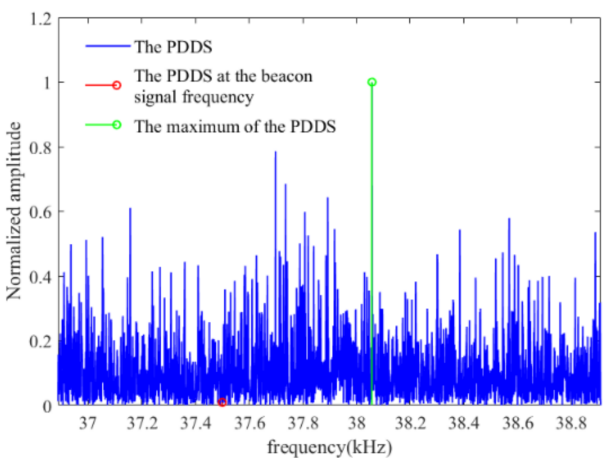

(c)

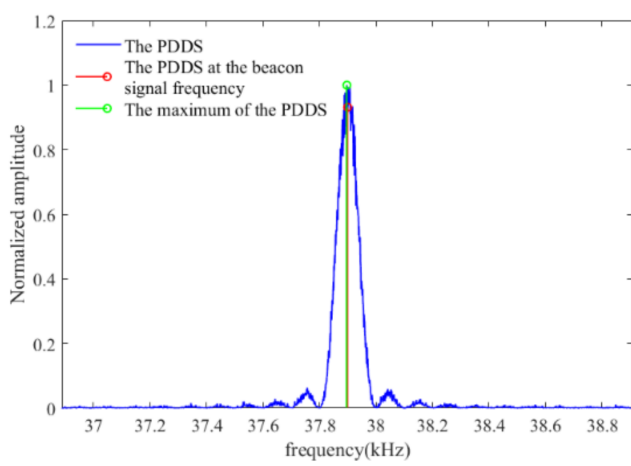

(b)

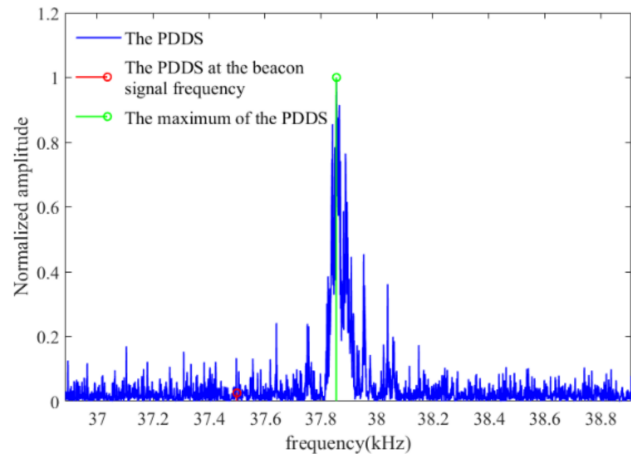

(d)

Figure 12. The PDDS without the random phase in various channels: (a) in the ideal channel with AWGN, (b) in the channel with AWGN and colored noise, (c) in the FFD channel with colored noise, and (d) in the TSD channel with colored noise.

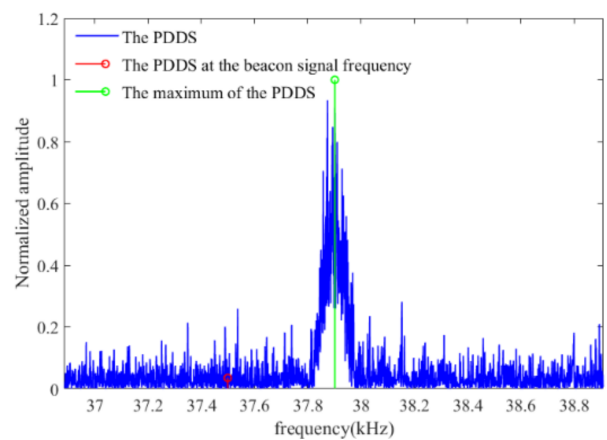

(a)

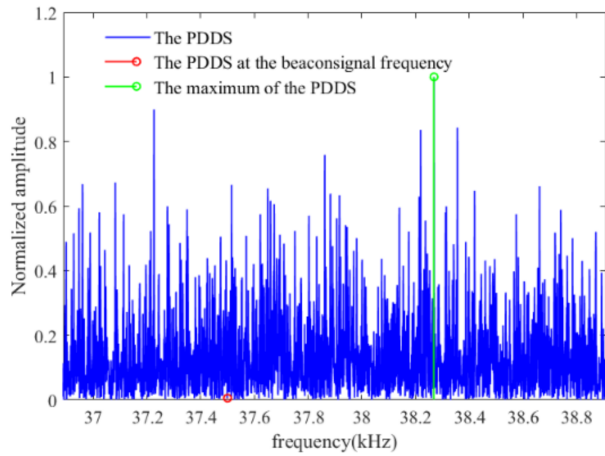

(c)

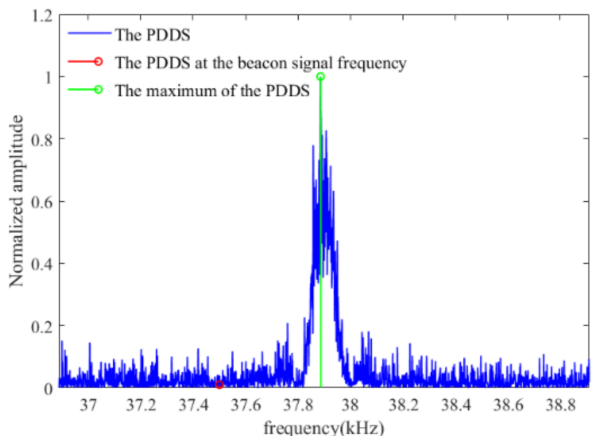

(b)

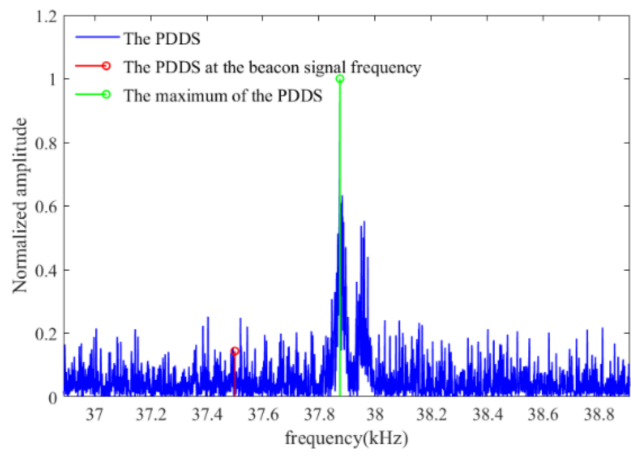

(d)

Figure 13. The PDDS with the random phase in various channels: (a) in the ideal channel with AWGN, (b) in the channel with AWGN and colored noise, (c) in the FFD channel with colored noise, and (d) in the TSD channel with colored noise. 


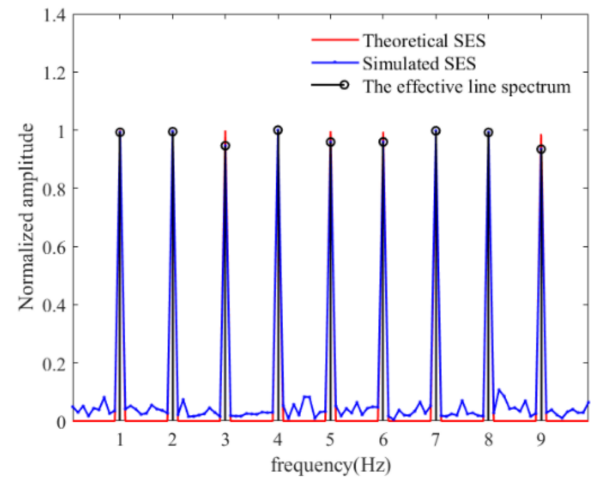

(a)

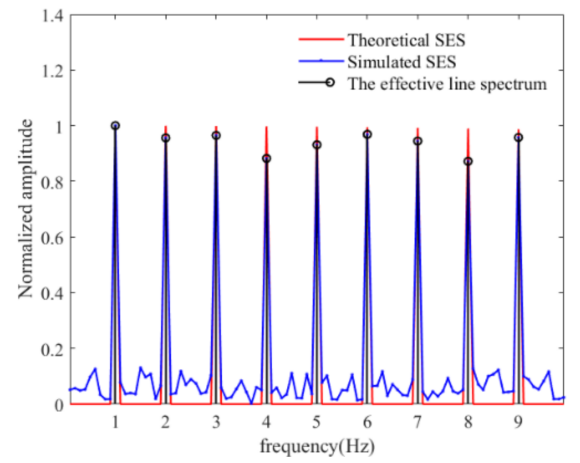

(c)

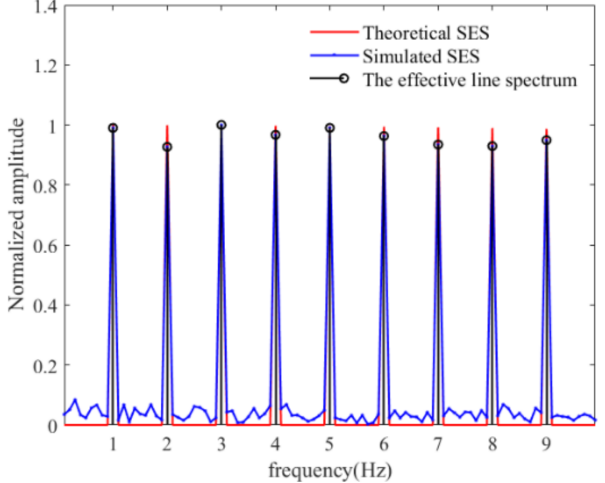

(b)

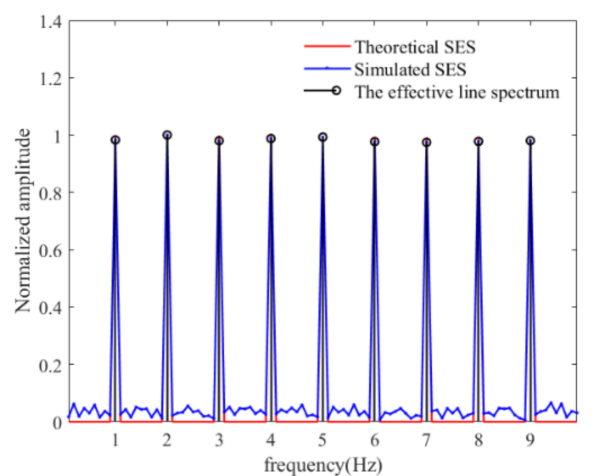

(d)

Figure 14. The SES without the random phase in various channels: (a) in the ideal channel with AWGN, (b) in the channel with AWGN and colored noise, (c) in the FFD channel with colored noise, and (d) in the TSD channel with colored noise.

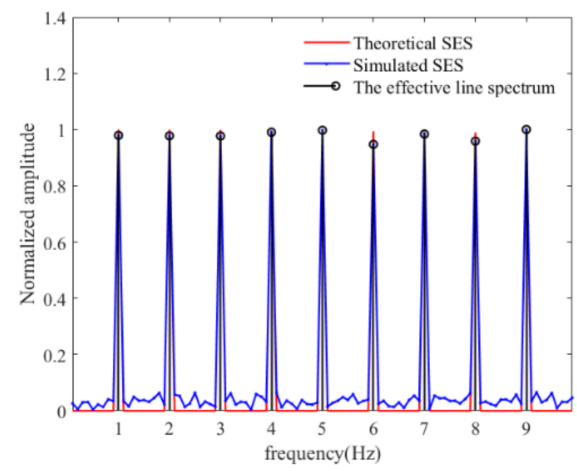

(a)

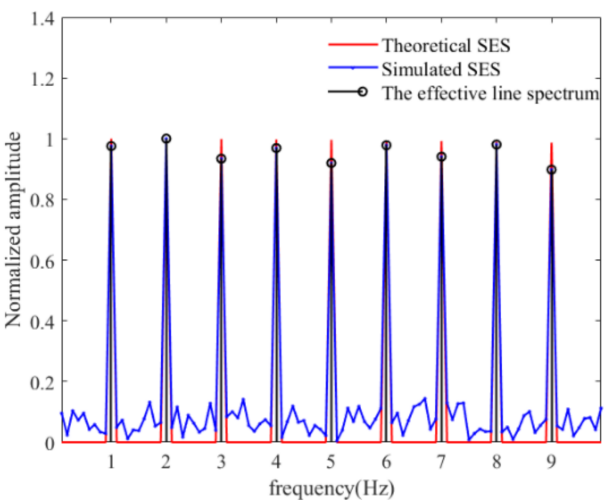

(c)

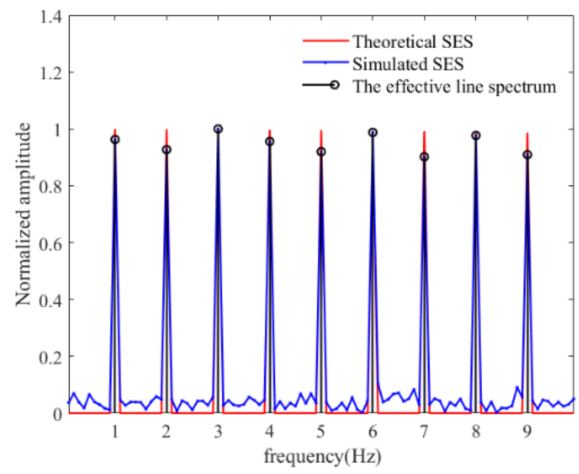

(b)

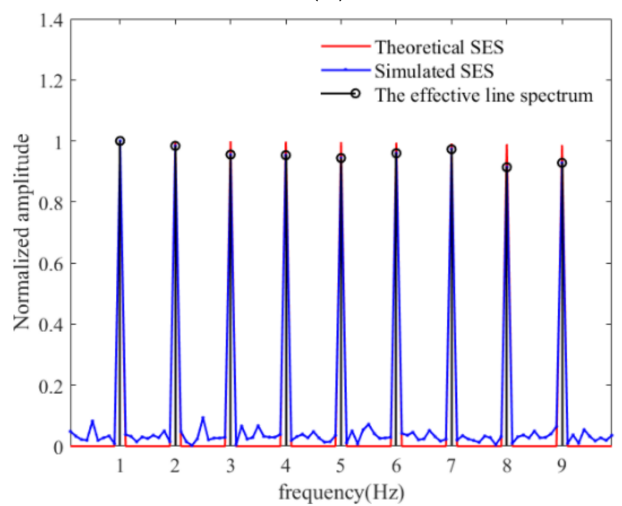

(d)

Figure 15. The SES with the random phase in various channels: (a) in the ideal channel with AWGN, (b) in the channel with AWGN and colored noise, (c) in the FFD channel with colored noise, and (d) in the TSD channel with colored noise. 
Figures 12 and 13, respectively, show the PDDS without the random phase and with the random phase in various channels. From Figures 12 and 13, we note that in the absence of the random phase, the effect of the PDDS is obvious with a high gain. However, the directly superposition method shows performance degradation in the presence of the random phase. It is caused by the fact that when there exists a random phase, sub-pulses for superposition cannot be aligned perfectly, and the gain of the DDS method is reduced. In Figures 12c and 13c, periodogram results show extremely poor performance in the FFD channel.

Figures 14 and 15, respectively, present the SES without the random phase and with the random phase in various channels. By contrast, the SES shows high robustness in all of the four cases, along with obvious characteristics of the line spectrum. Thus, considering the desirable properties of SES in various channels, the SES method is the preferred method of the two herein, and, as predicted by theory, the SES overcomes the problem of the random phase with identical test results.

Case 3: Detection performance in various channels with Doppler shift

We tested the superiority of the proposed detector and other well-known methods by drawing the detection probability curves. Specifically, Monte Carlo simulations with 1000 runs are used. In each simulation, the SNR ranges from $-25 \mathrm{~dB}$ to $15 \mathrm{~dB}$ in a step of $2 \mathrm{~dB}$, and the false alarm probability $P_{f}$ is set to $1 \%$. For each experiment, the MF, GLRT, and PDDS detectors are presented for comparison. Figures 16-19 show the detection probability $P_{d}$ of each method in various channels for beacon signal detection. The detection performance is measured by relative SNR required for a $P_{d}$ of $90 \%$.

Figure 16 shows the detection curves in the ideal channel with AWGN. It is noted that the PDDS detector without the random phase performs the best, followed by the MF detectors and the PDDS detector with the random phase. The proposed detector performs slightly worse and the GLRT detector performs the worst. In detail, the proposed detector requires an SNR of $-6 \mathrm{~dB}$ to achieve a $P_{d}$ of $90 \%$, whose performance loss relative to the best detector is $17 \mathrm{~dB}$, and the performance gain relative to the worst detector is $3 \mathrm{~dB}$.

In Figure 16, the PDDS detector is significantly affected by the random phase. Its performance without the random phase is excellent, but the random phase brings about a performance loss of $8 \mathrm{~dB}$ in SNR. However, it is more common to operate the detector in an environment with the random phase. For the proposed detector, the detection performances with or without the random phase are approximately consistent. The proposed detector requires an SNR of $-6 \mathrm{~dB}$ to achieve a detection probability of $90 \%$, whose performance loss relative to the best detector is about $21 \mathrm{~dB}$ in term of $P_{d}=90 \%$, and the performance gain relative to the worst detector is about $3 \mathrm{~dB}$.

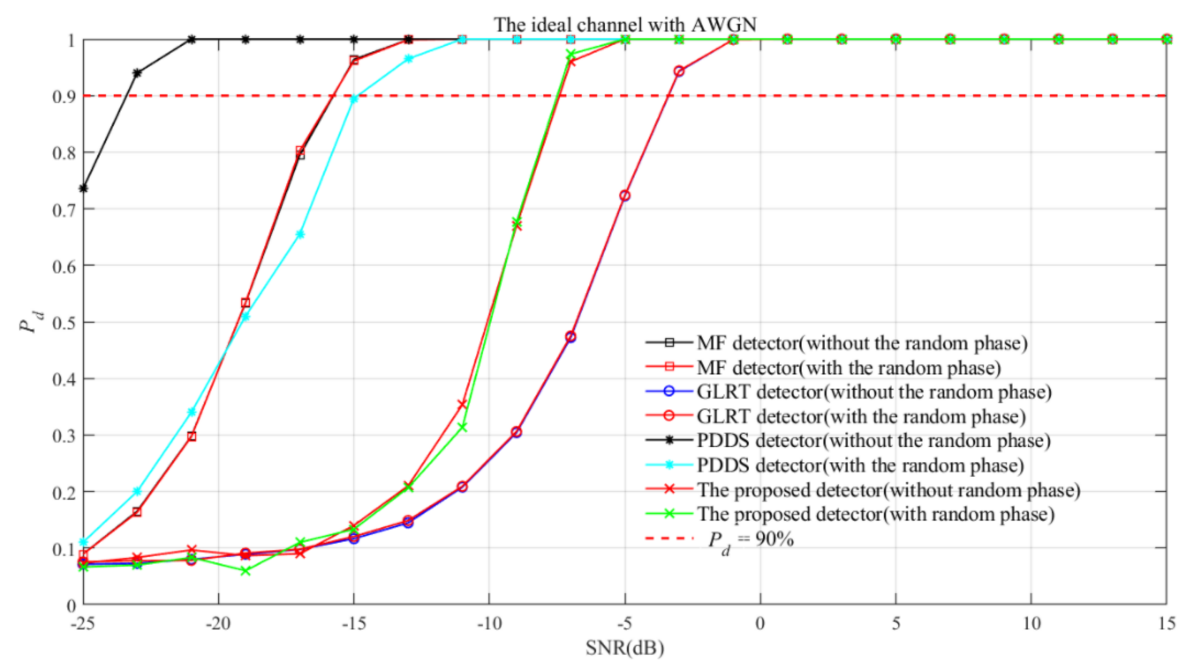

Figure 16. Detection probability $P_{d}$ vs. SNRs in the ideal channel with AWGN. 
Figure 17 shows the results in a channel with colored noise and AWGN. From Figure 17, it is noticeable that the PDDS detector is still the best in the absence of the random phase, followed by the MF detector and the PDDS detector with random phase. The GLRT detector performs worst. Compared to the ideal channel with AWGN, as shown by Figure 16, the performance in the channel with colored noise and AWGN for the conventional detectors decreases $0.3-1 \mathrm{~dB}$. In terms of the relative SNR required for a $P_{d}$ of $90 \%$, the proposed detector has a performance loss of about $15 \mathrm{~dB}$ relative to the best detector and has a performance gain of $5 \mathrm{~dB}$ relative to the worst detector. Similarly, the PDDS is negatively affected by the random phase, and the other three detectors are barely influenced.

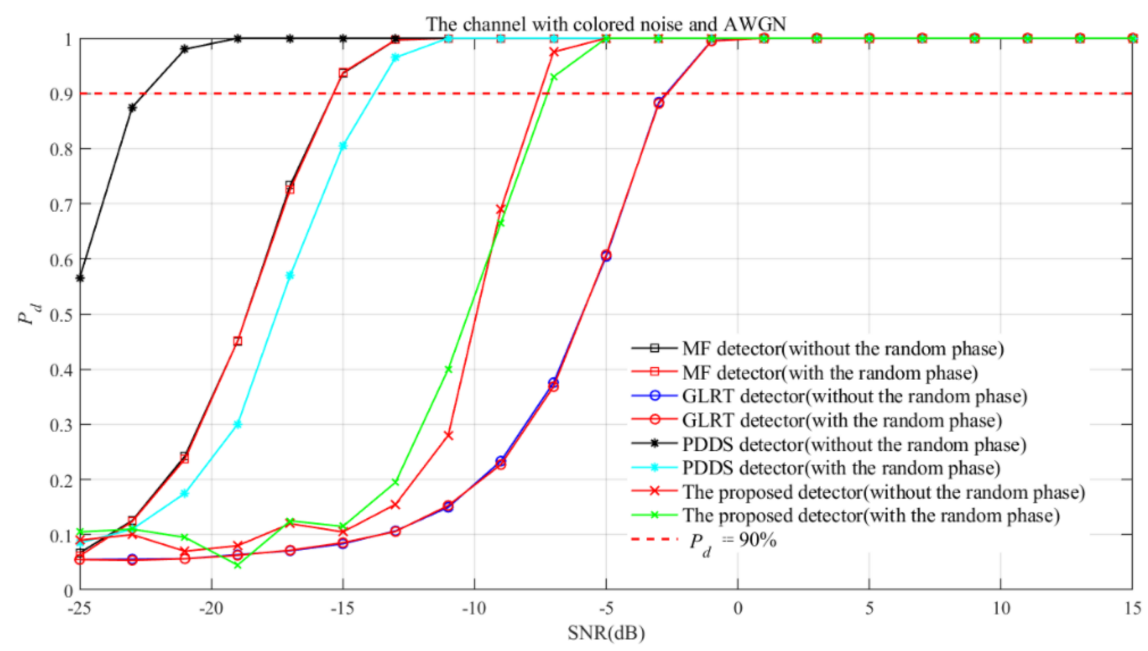

Figure 17. Detection probability $P_{d}$ vs. SNRs in the channel with colored noise and AWGN.

Now, consider a channel with colored noise and distortions. Figure 18 shows the performance of the detectors in the FFD channel with colored noise. It is interesting to note that the proposed detector performs the best, while all other compared conventional detectors perform quite poorly. Among the conventional ones, the MF detector is slightly better, the PDDS detector without the random phase is poorer, and the GLRT and the PDDS detectors with the random phase are the worst. In detail, the proposed detector shows performance gains of about $12 \mathrm{~dB}, 13.6 \mathrm{~dB}, 16.6 \mathrm{~dB}$, and $16.6 \mathrm{~dB}$ relative to the MF detector, PDDS detector without random phase, GLRT detector, and PDDS detector with the random phase.

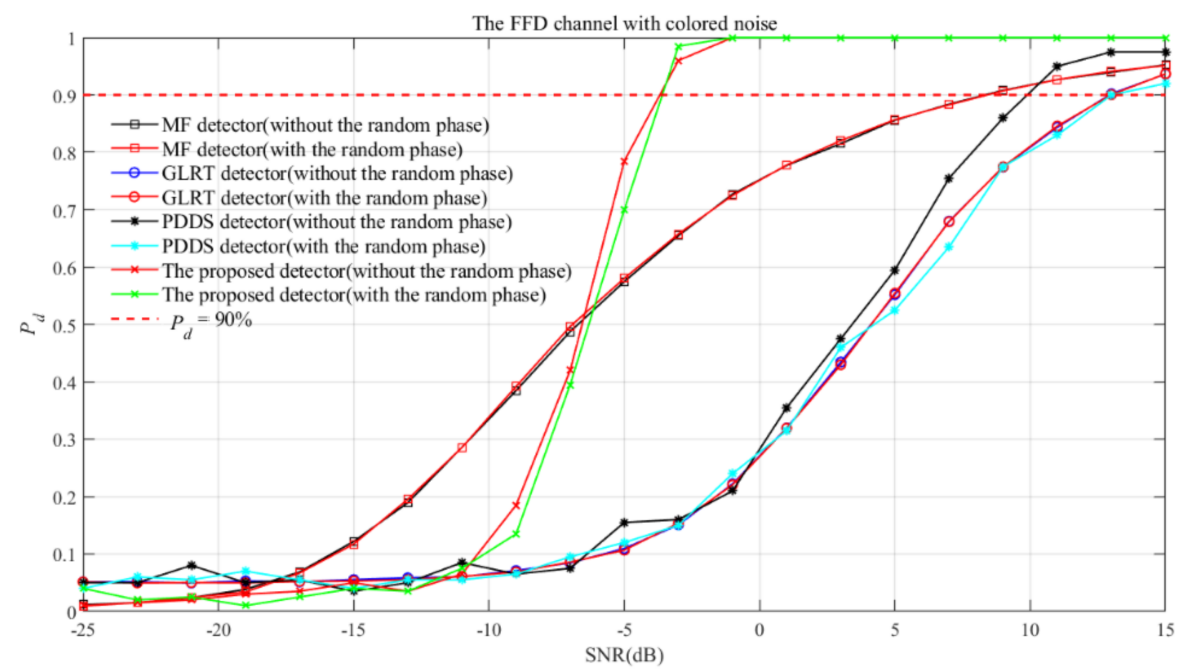

Figure 18. Detection probability $P_{d}$ vs. SNRs in the FFD channel with colored noise. 
Figure 19 shows the performance of detectors in the TSD channel with colored noise. The proposed detector is still the best one among the four herein, and the conventional detectors show poor performance affected by the distorted channel. The PDDS detector and GLRT detector are slightly worse, and the MF detector performs the worst. The proposed detector outperforms the PDDS, GLRT, and MF detectors by $3.3 \mathrm{~dB}, 3 \mathrm{~dB}$, and $3.3 \mathrm{~dB}$, respectively, measured by the relative SNR required for a $P_{d}$ of $90 \%$.

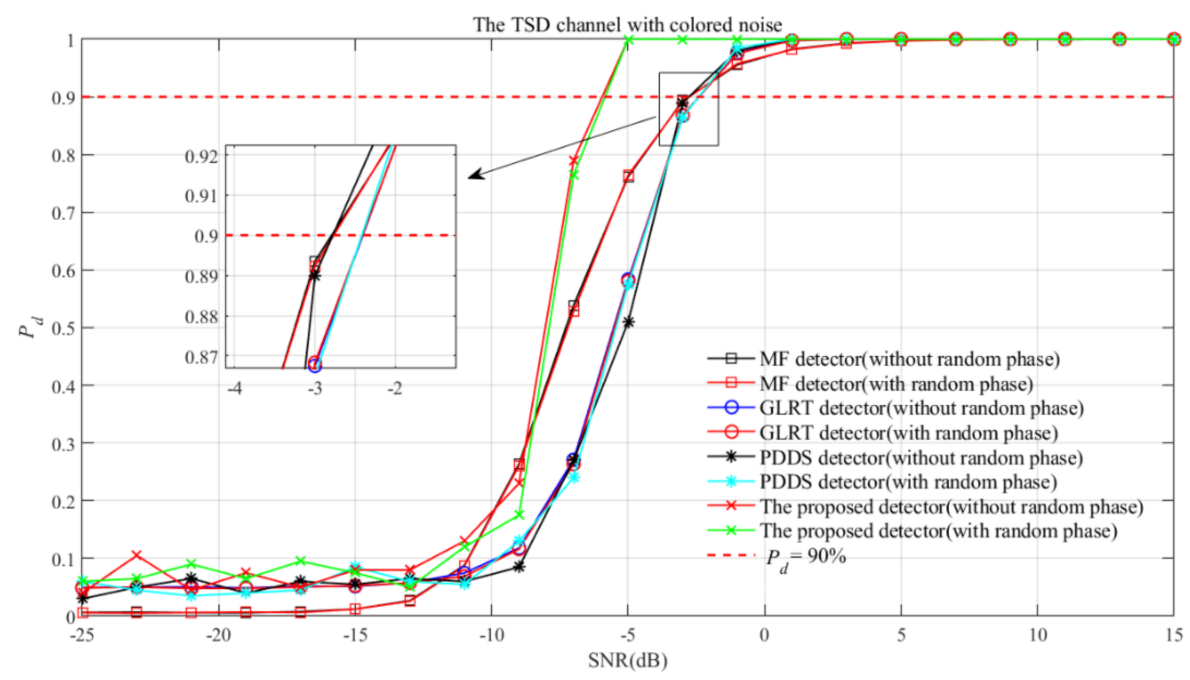

Figure 19. Detection probability $P_{d}$ vs. SNRs in the TSD channel with colored noise.

In the TSD channel, the impacts of the random phase are not obvious. In other words, the detection performance for each detector is similar with or without the random phase. This is because the TSD effects change the phase of the received signal.

In sum, the tests above demonstrate the robustness and great performance of the proposed detector. On the one hand, Figures 16 and 17 suggest that the proposed detector is robust against the random phase. In the channel with AWGN and colored noise, the random phase significantly reduces the detection probability $P_{d}$ of the PDDS detector, but it has almost no effect on the proposed detector. On the other hand, the proposed detector shows good robustness in the distorted channels. Figures 18 and 19 suggest that the proposed detector outperforms other detectors in the presence of complicated channel distortions FFD and TSD. From Table 1, it is easy to see that, in terms of the relative SNR required for a $P_{d}$ of $90 \%$ in various channels, the detection performance of the proposed detector is hardly affected by the colored noises and distortions, verifying its advantage over the other three detectors.

Table 1. SNRs $(\mathrm{dB})$ of the various detection methods with random phase given a $P_{d}$ of $90 \%$ and a $P_{d}$ of $1 \%$.

\begin{tabular}{|c|c|c|c|c|}
\hline Detector & $\begin{array}{c}\text { MF } \\
\text { Detector }\end{array}$ & $\begin{array}{c}\text { GLRT } \\
\text { Detector }\end{array}$ & $\begin{array}{c}\text { PDDS } \\
\text { Detector }\end{array}$ & $\begin{array}{c}\text { The Proposed } \\
\text { Detector }\end{array}$ \\
\hline AWGN & -15.8 & -3.4 & -14.8 & -7.5 \\
\hline Colored noise and AWGN & -15.4 & -2.7 & -13.8 & -7.2 \\
\hline FFD with colored noise & 8.4 & 13 & 13 & -3.6 \\
\hline TSD with colored noise & -2.8 & -2.5 & -2.5 & -5.8 \\
\hline
\end{tabular}

\subsection{Experimental Validation Based on Real Lake Trial Data}

The simulation results show that the proposed detector outperforms the existing stateof-the-art detectors in channels with various distortions and colored noise. To validate the feasibility of the proposed detector, we conducted experiments with real lake trial data. The lake trial was carried out at the Geheyan Reservoir area in Hubei province of China $\left(111.12^{\circ} \mathrm{N}, 30.42^{\circ} \mathrm{E}\right.$ ) on 21 December 2020 (as shown in Figure 20). 


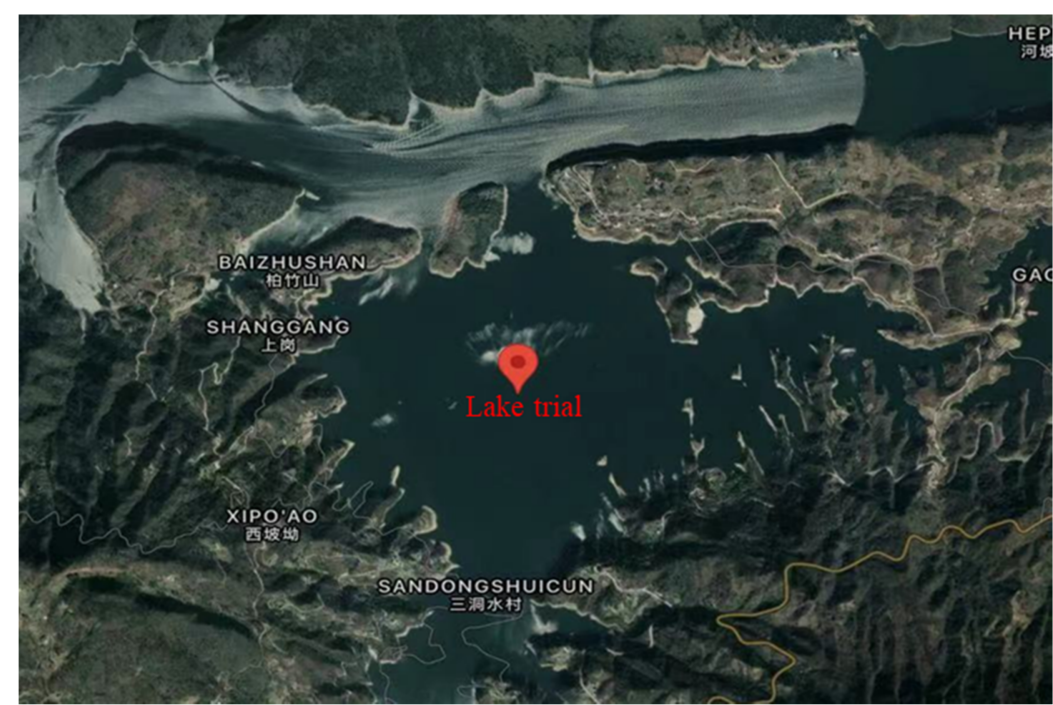

Figure 20. Experiment test site conducted at Geheyan Reservoir, Hubei, China, on 21 December 2020.

The schematic diagram of the lake trial is shown in Figure 21. As shown in Figure 21, both the underwater acoustic beacon and the receiving hydrophone are about $20 \mathrm{~m}$ to the water surface. The underwater acoustic beacon (type DKM590) was mounted on a small boat which anchored at the appointed position. The distance between the underwater acoustic beacon and the receiving hydrophone is approximately $3 \mathrm{~km}$. The receiving hydrophone was mounted on an unmanned underwater vehicle (UUV), and the UUV moved in a circle centered on the small boat at a speed of 4 knots. The propagation speed of the sound in the lake trial area was about $1490 \mathrm{~m} / \mathrm{s}$.

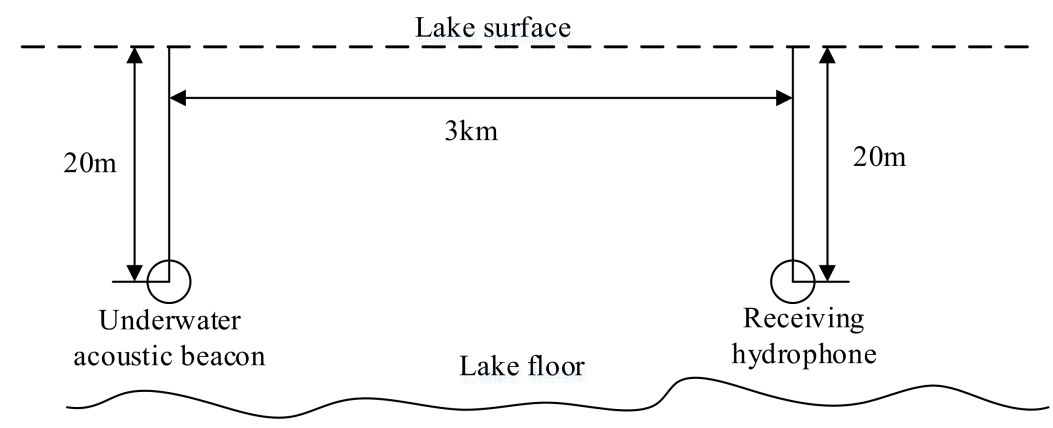

Figure 21. Schematic diagram of the lake trial.

Now, we consider the real lake trial data which contains multi-pulse beacon signal to verify the application of the proposed detector for detection of the beacon signal using multi-pulses. The parameters of the real lake trial data are the same as presented in Section 2.1 (i.e., $\tau=10 \mathrm{~ms}, T=1 \mathrm{~s}$, and $f_{0}=37.5 \mathrm{kHz}$ ). The number of the received pulses $N$ is 100. The number of reserved pulses after aligned superimposition process $K$ is 10 and the times of superposition $L$ is 10 . Similarly, we compare the results of the MF detector, GLRT detector, PDDS detector, and the proposed detector.

Figure 22a,b show the time series and the time-frequency distribution (TFD) of the received lake trial data. In Figure 22a, the signal part cannot be distinguished from the time series due to noise and interference. In Figure 22b, the properties of $1 \mathrm{~s}$ period and $37.5 \mathrm{kHz}$ frequency are clear. However, from the partially enlarged part of Figure 22, it can be seen that the received signals show serious time extension; this is called the TSD effect in this paper. Besides, there are some energy fluctuations immediately following the signal part. The SNRs of the received pulses vary between $-6 \mathrm{~dB}$ and $3 \mathrm{~dB}$. 


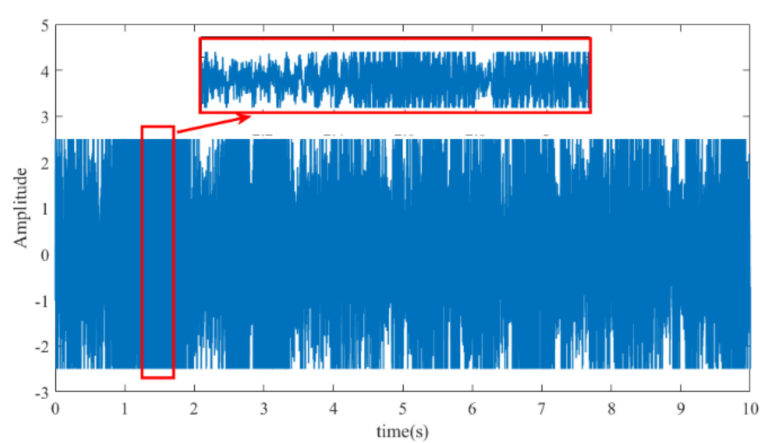

(a)

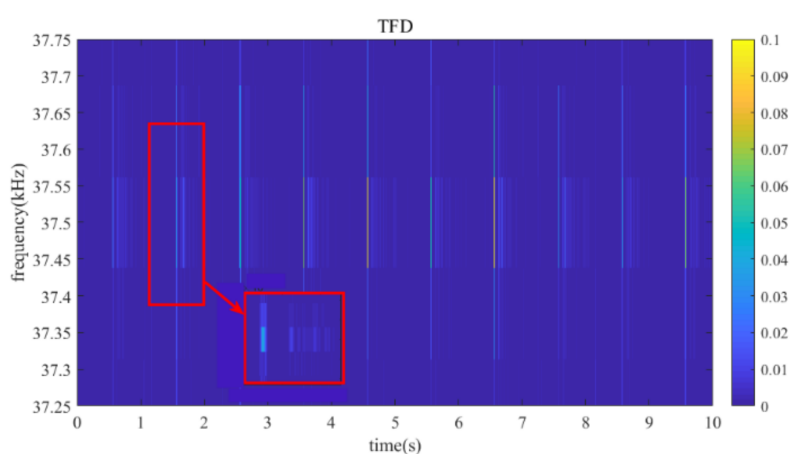

(b)

Figure 22. The time series and time-frequency distribution (TFD) of the received lake trial data. (a) The time series, (b)The time-frequency distribution (TFD).

Figure 23 shows the processed results. In Figure 23, the MF detector output is shown. From Figure 23, it is noticeable that peaks at the location of pulses are clear, and each pulse varies in peak amplitude. Specifically, when the threshold is set to 0.4 , six pulses can be detected, but when the threshold is set to 0.7, only two pulses can be detected. The detection result depends largely on the threshold. Thus, for the MF detector, a major concern is that it is difficult to set a reasonable detection peak value threshold to obtain stable and effective detection results.

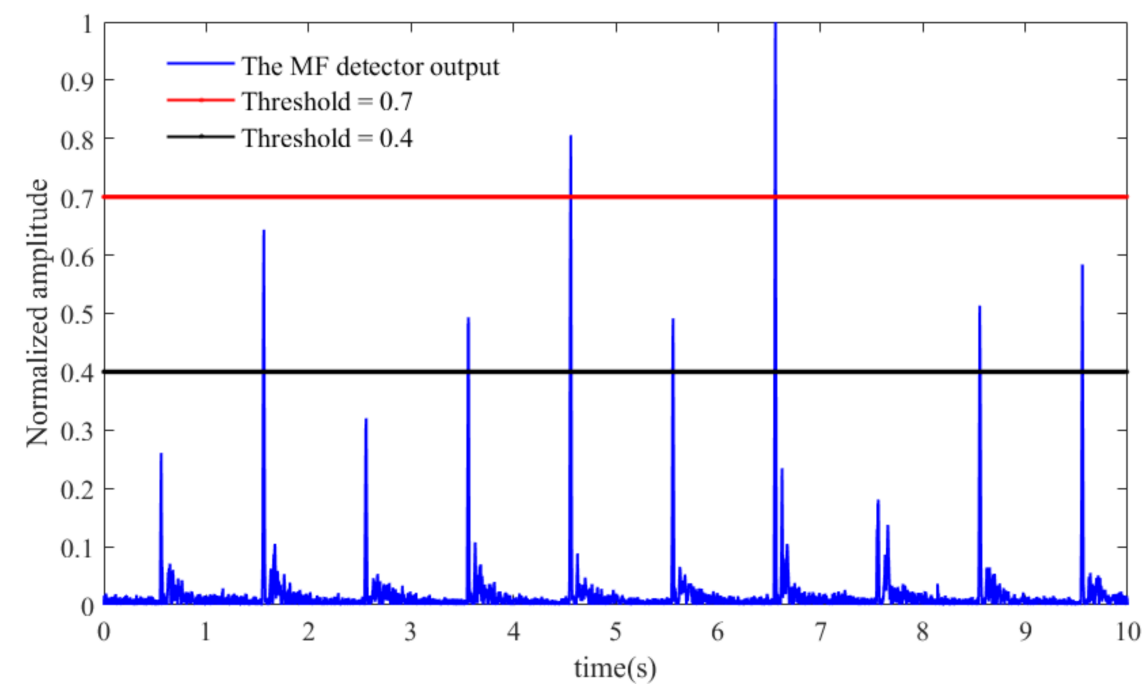

Figure 23. The MF detector output of the received lake trial data.

Figure 24a-f show the results of the GLRT detector, which are normalized local periodograms of six pulses of the received lake trial data. They are the judgment basis of the GLRT detector. Figure 24a-f present the normalized local periodogram near $37.5 \mathrm{kHz}$. The observed frequency range is $37.25 \mathrm{kHz}$ to $37.75 \mathrm{kHz}$. We zoom in on regions near the known signal frequency $(37.5 \mathrm{kHz})$ and locally normalize the spectrum. The signal energy can be observed. Note that the energy is diffused, instead of concentrated on a single frequency point. Besides, the periodogram at $37.5 \mathrm{kHz}$ is not the maximum of the periodogram. In Figure $25 \mathrm{c}$, the periodogram at $37.5 \mathrm{kHz}$ is even lower than the average of the local normalized periodogram. These phenomena are the results of the Doppler effect and distortions, and they will lead to the decrease in the detection performance. 


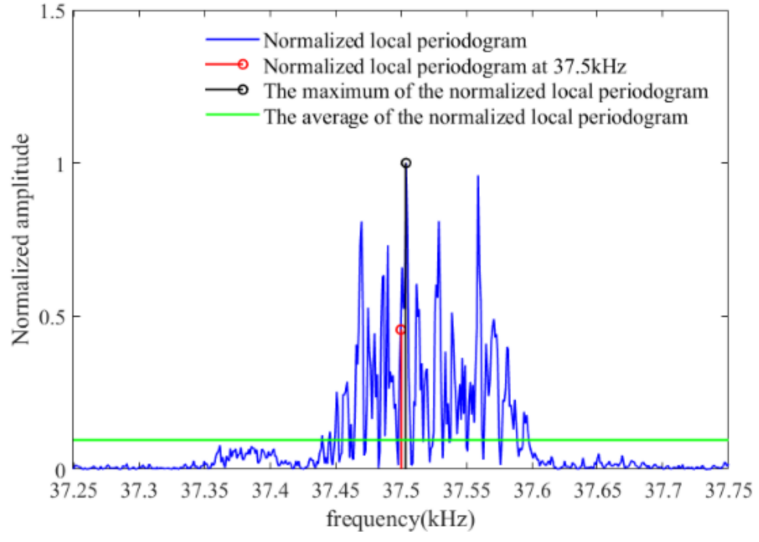

(a)

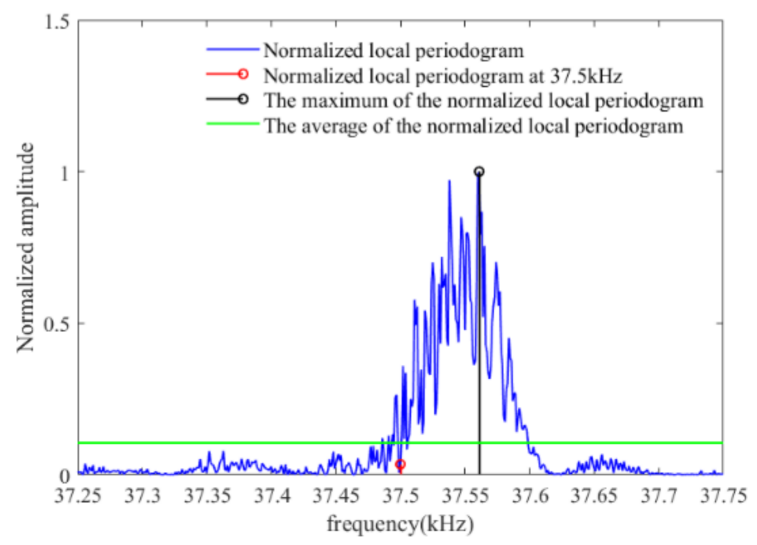

(c)

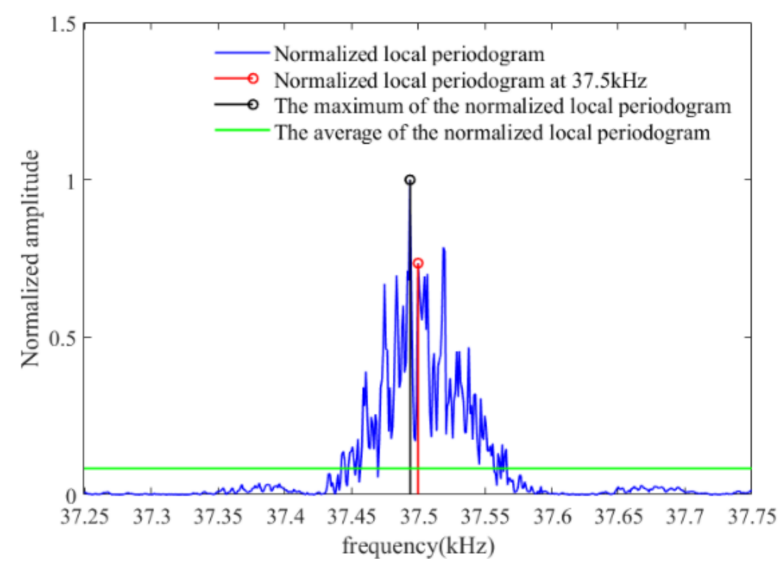

(e)

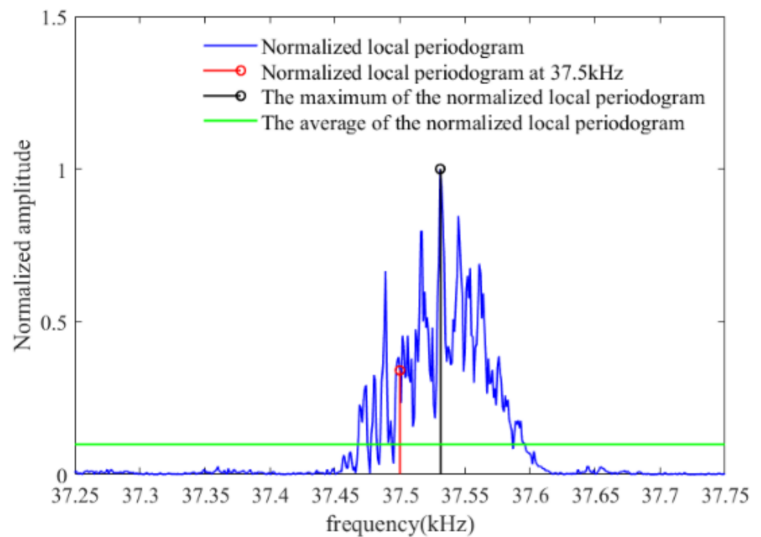

(b)

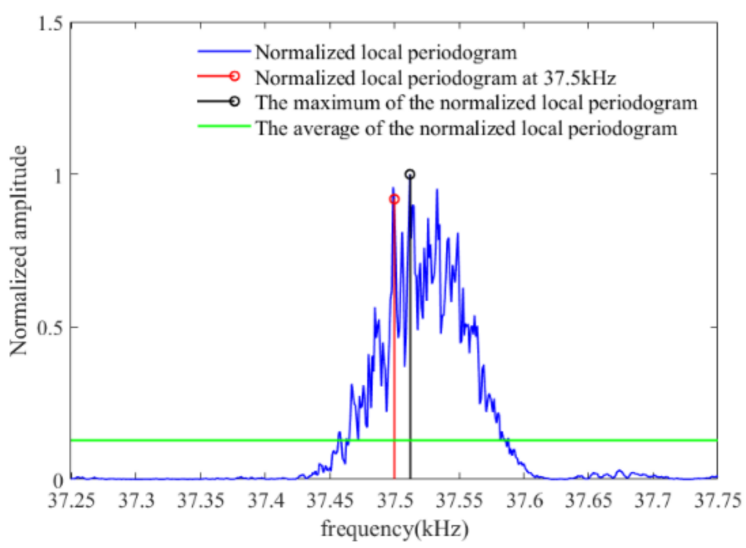

(d)

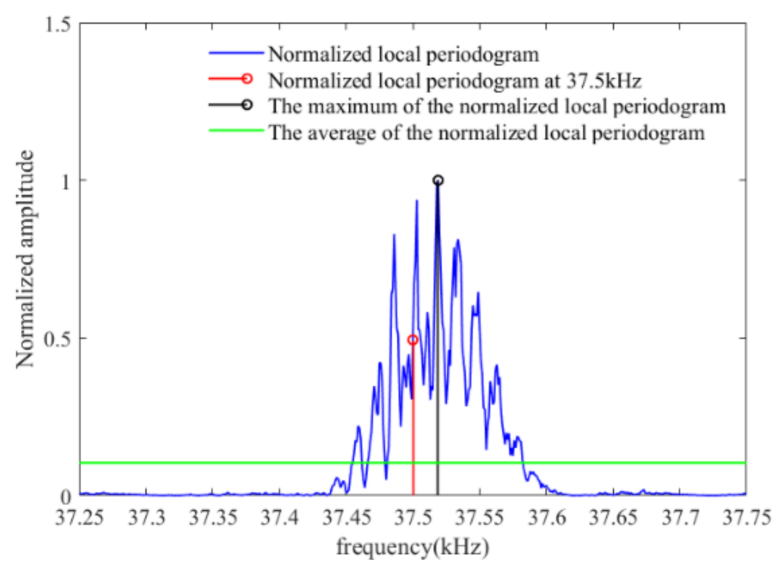

(f)

Figure 24. Normalized local periodogram of single pulse of received lake trial data. The normalized local periodogram of (a) pulse 1, (b) pulse 2, (c) pulse 3, (d) pulse 4, (e) pulse 5, and (f) pulse 6.

Figure 25 shows the results of the PDDS detector. To analyze the gain, we calculate the average of periodograms of 10 pulses and normalize it locally at near $37.5 \mathrm{kHz}$. For simplified analysis, the PDDS is also normalized locally near $37.5 \mathrm{kHz}$, and it is referred to as normalized local PDDS. From Figure 25, it can be noted that the PDDS obtains a processed gain compared with the average of periodograms of 10 pulses. Still, the spectrum diffusion exists in normalized local PDDS, and the detection results are limited by the set threshold. 


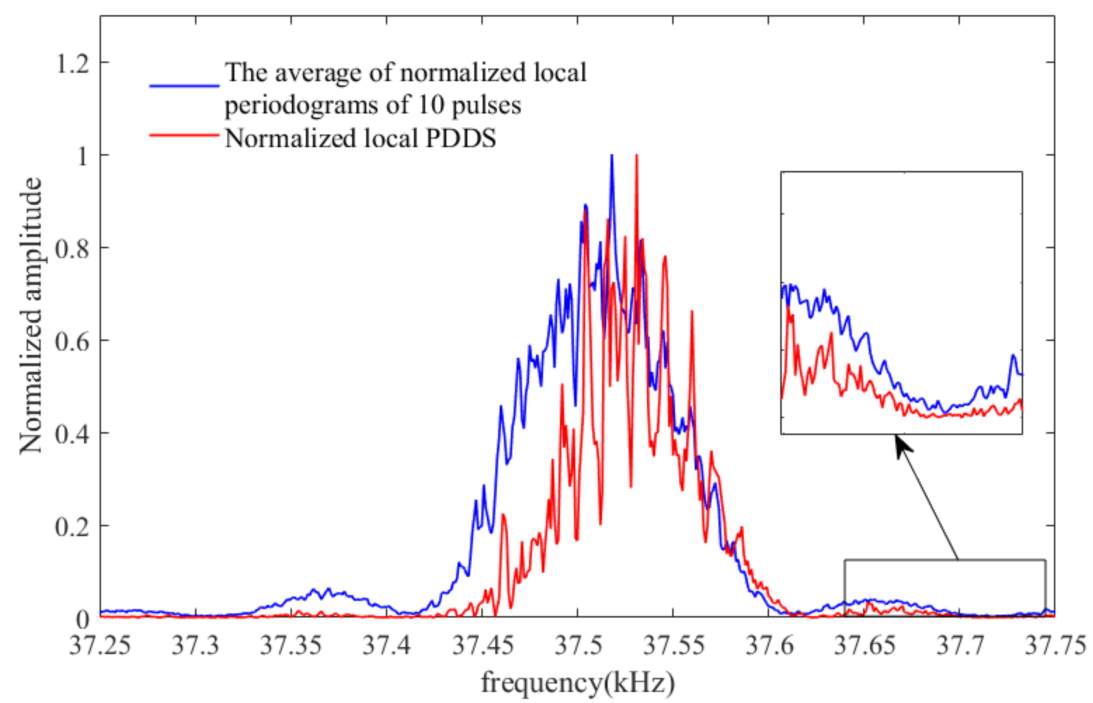

Figure 25. Normalized local PDDS and the average of normalized periodograms of 10 pulses of received lake trial data.

Now, let us compare the PDDS and the proposed superimposed envelope. As mentioned before, the former is a superposition of the received signal waveform, and the latter is the superposition of the extracted envelope. It is expected, in theory, that the superimposed envelope has a higher gain and better robustness. Figure 26a,b show the superimposed signal and envelopes of the received lake trial data. Similarly, we normalized the amplitude of the two. From Figure 26a, it can be seen that for the superimposed signal, the signal part is hard to distinguish from the time series. In Figure 26b, the superimposed envelope, by comparison, has a more obvious amplitude fluctuation than the noise part. Figure $26 \mathrm{~b}$ compares the envelopes and the superimposed envelopes of the received lake trial data. The superimposed envelopes perform well with a high gain and great robustness against misalignment. During the superimposition process, the signal part in one period is greatly increased. In this case, it is easy to determine the position of the signal pulse from the envelope. Besides, with such obvious and robust periodicity, it helps the detection system with the following analysis and detection.

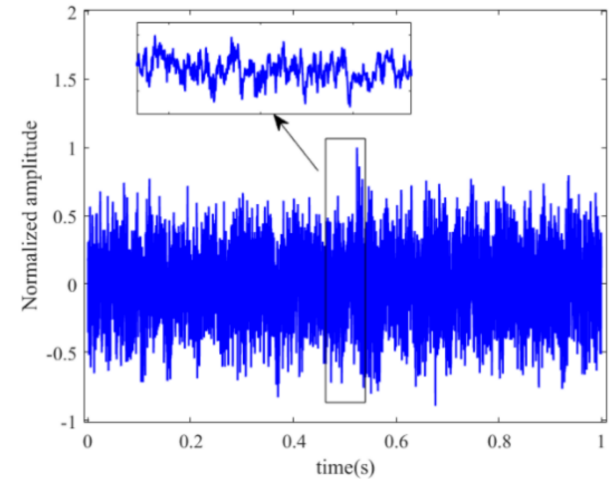

(a)

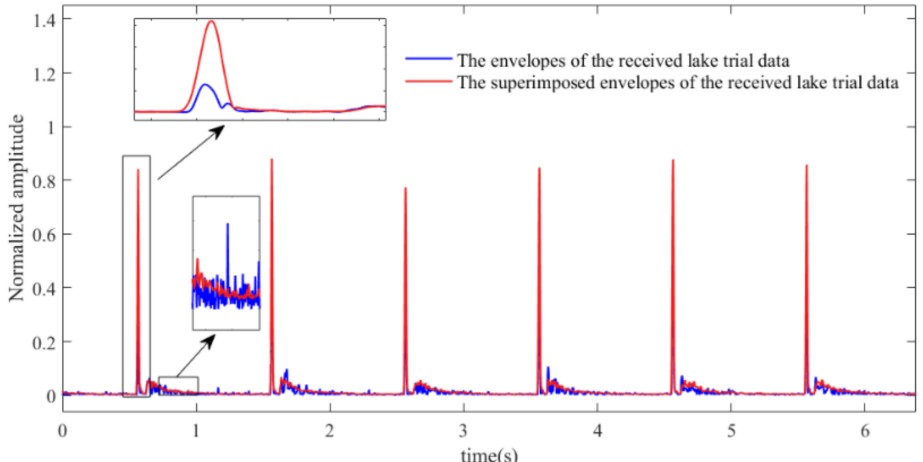

(b)

Figure 26. The superimposed signal and the superimposed envelope of the lake trial data. (a) The superimposed signal in time domain. (b) The superimposed envelope in time domain.

When applying the superimposed envelope in the further detection process, we should also consider the TSD effect, which may lead to some amplitude fluctuations following the signal part. From Figure 26b, we note that the amplitude of the fluctuations is much lower than the signal part, which would not influence the SES-based detection. 
Eventually, the results of the proposed detector are illustrated in Figure 27a,b. Figure $27 \mathrm{a}, \mathrm{b}$ present the theoretical SES, the envelope spectrum (ES) of lake trial data, the SES of lake trial data, and the effective spectral lines of two sets of received lake trial data. To analyze the superimposed gain, the ES of lake trial data with 10 pulses is shown in Figure 27. From Figure 27, it can be seen that the locations of the spectral lines of the SES are the same as those of the ideal SES, and, compared to the ES, the spectral lines of the SES have higher LSNR. With such robust and clear periodic peak characteristics, it is easy to detect the line spectrum using the decision-making method in Section 3.2.2.

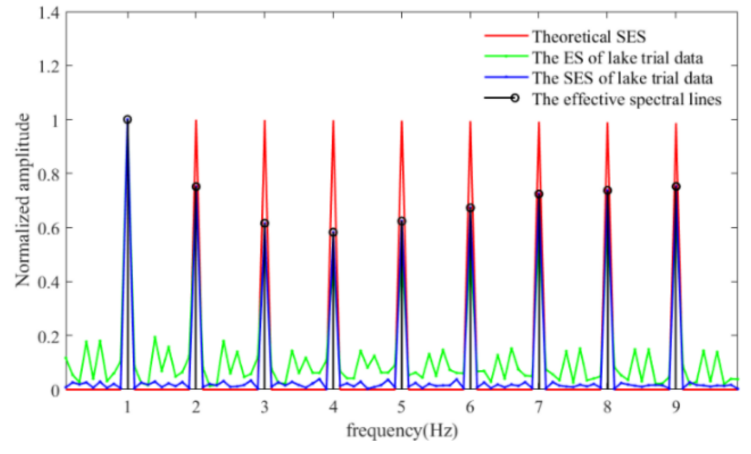

(a)

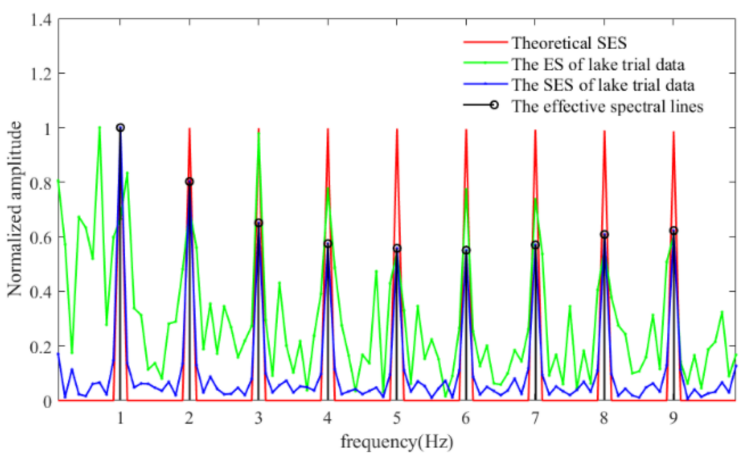

(b)

Figure 27. Theoretical SES, the ES of lake trial data, the SES of lake trial data, and the effective spectral lines. (a) The results of the first set of the received lake trial data. (b) The results of the second set of the received lake trial data.

\section{Conclusions}

The underwater beacon signal suffers from colored noise and various distortions propagating through the underwater channel. Various noise and distortions are considered in this paper, including the AWGN, the colored noise, and the distorted channel. We propose a novel detector based on the features of the SES for detecting the underwater acoustic beacon signal in various noisy and distorted channels. The superimposed envelope enhances the part of the beacon signal and obtains the process gain. The characteristics of the SES (comb-shaped, harmonic, locally periodic, and convergent) keep constant with either aligned superimposition or not. According to the characteristics of the SES, a judgement is designed based on the extracted effective spectral lines. Unlike the conventional methods, which are sensitive to the colored noise, the random phase, or the channel distortions, the proposed SES method is robust against them. Simulation results show that, for a given false alarm probability of $1 \%$ and a detection probability value of $90 \%$, the proposed detector based on the SES outperforms the conventional detectors with a gain of 3-12 dB in terms of the relative SNR under distorted channels, and the experimental results based on the real lake trial data verified the validity of the proposed detector. Therefore, among the four detectors, the proposed method has the best performance in terms of robustness as well as the SNR gain, while the MF is extremely poor in the UWAN. In future work, we will focus on obtaining a higher SNR gain for the proposed detector by combining it with array processing technologies.

Author Contributions: Conceptualization, S.Y.; Funding acquisition, S.Y.; Investigation, S.Y.; Methodology, S.Y. and Y.L.; Software, S.Y. and Y.L.; Supervision, S.Y.; Writing-original draft, Y.L.; Writingreview and editing, S.Y. and Y.L. All authors have read and agreed to the published version of the manuscript.

Funding: This research was funded in part by the National Natural Science Foundation of China, grant number 11704069, 11874109 and 12174053, and was supported in part by the Fundamental Research Funds for the Central Universities under Grant No. 2242021k30019.

Institutional Review Board Statement: Not applicable. 
Informed Consent Statement: Not applicable.

Data Availability Statement: Not applicable.

Acknowledgments: The authors would like to thank the anonymous reviewers for their useful comments and suggestions.

Conflicts of Interest: The authors declare no conflict of interest.

\section{References}

1. Mujeebu, M.A. The disappearance of MH370 and the search operations-The role of technology and emerging research challenges. IEEE Aerosp. Electron. Syst. Mag. 2016, 31, 6-16. [CrossRef]

2. Mcnutt, M. The hunt for MH370. Science 2014, 344, 947. [CrossRef] [PubMed]

3. Stone, L.D.; Keller, C.M.; Kratzke, T.M.; Strumpfer, J.P. Search for the Wreckage of Air France Flight AF 447. Stat. Sci. 2014, 29, 69-80. [CrossRef]

4. Basagni, S.; Conti, M.; Giordano, S.; Stojmenovic, I. Advances in Underwater Acoustic Networking; John Wiley \& Sons, Inc.: Hoboken, NJ, USA, 2013; pp. 804-852.

5. Kay, S. Fundamentals of Statistical Signal Processing, Volume II: Detection Theory; PTR Prentice Hall: Englewood Cliffs, NJ, USA, 1993.

6. Levy, B.C. Principles of Signal Detection and Parameter Estimation; Springer Publishing Company, Incorporated: Berlin/Heidelberg, Germany, 2008.

7. Baggenstoss, P.M.; Kay, S.M. An adaptive detector for deterministic signals in noise of unknown spectra using the Rao test. IEEE Trans. Signal Process. 1992, 40, 1460-1468. [CrossRef]

8. Trees, H.L.V. Detection, Estimation, and Modulation Theory: Radar-Sonar Signal Processing and Gaussian Signals in Noise; Krieger Publishing Co., Inc.: Malabar, FL, USA, 1992.

9. Bouvet, M.; Schwartz, S.C. Comparison of adaptive and robust receivers for signal detection in ambient underwater noise. IEEE Trans. Acoust. Speech Signal Process. 1989, 37, 621-626. [CrossRef]

10. Chitre, M.; Potter, J.; Heng, O.S. Underwater acoustic channel characterisation for medium-range shallow water communications. Oceans 2004, 1, 40-45.

11. Chitre, M.; Kuselan, S.; Pallayil, V. Ambient noise imaging in warm shallow waters; robust statistical algorithms and range estimation. J. Acoust. Soc. Am. 2012, 132, 838-847. [CrossRef] [PubMed]

12. Stojanovic, M.; Preisig, J. Underwater acoustic communication channels: Propagation models and statistical characterization. IEEE Commun. Mag. 2009, 47, 84-89. [CrossRef]

13. Legg, M.W. Non-Gaussian and Non-Homogeneous Poisson Models of Snapping Shrimp Noise. Ph.D. Dissertation, Curtin University of Technology, Perth, Australia, 2010.

14. Mahmood, A.; Chitre, M. Optimal and Near-Optimal Detection in Bursty Impulsive Noise. IEEE J. Ocean. Eng. 2017, 42, 639-653. [CrossRef]

15. Al-Aboosi, Y.; Sha'Ameri, A.; Khamis, N. Comparison of methodologies for signal detection in underwater acoustic noise in shallow tropical waters. J. Eng. Appl. Sci. 2016, 11, 3086-3094.

16. Al-Aboosi, Y.Y.; Sha'Ameri, A.Z. Improved underwater signal detection using efficient time-frequency de-noising technique and Pre-whitening filter. Appl. Acoust. 2017, 123, 93-106. [CrossRef]

17. Guo, G.; Mandal, M.; Jing, Y. A robust detector of known signal in non-Gaussian noise using threshold systems. Signal Process. 2012, 92, 2676-2688. [CrossRef]

18. Baggenstoss, P.M. On detecting linear frequency-modulated waveforms in frequency- and time-dispersive channels: Alternatives to segmented replica correlation. IEEE J. Ocean. Eng. 1994, 19, 591-598. [CrossRef]

19. Friedlander, B.; Zeira, A. Detection of broadband signals in frequency and time dispersive channels. IEEE Trans. Signal Process. 1996, 44, 1613-1622. [CrossRef]

20. Zhang, Z.; Cheng, X.; Ning, D.; Hou, J.; Gong, Y. Underwater acoustic beacon signal extraction based on dislocation superimposed method. Adv. Mech. Eng. 2017, 9, 1687814017691671. [CrossRef]

21. Yao, S.; Yu, W.; Fang, S.; Huang, S. Robust Active Sonar Detection in Frequency and Time Dispersive Channels Using Matching Envelope Spectrum of Multi-Pulse LFM Signals. IEEE Access 2020, 8, 159990-160003. [CrossRef]

22. Wu, Q.; Xu, P.; Li, T.; Fang, S. Feature Enhancement Technique with Distorted Towed Array in the Underwater Radiated Noise. In Proceedings of the INTER-NOISE and NOISE-CON Congress and Conference Proceedings, Hong Kong, China, 27-30 August 2017; Institute of Noise Control Engineering: Washington, DC, USA, 2017.

23. Tao, D. Recognizing ship objects by the shape of averaged power spectrum of radiated noise. Acta Acust. 1981, 4, 219-228. (In Chinese)

24. Zhu, C.; Fang, S.; Wu, Q.; An, L.; Luo, X.; Cao, H. A Time-Frequency Joint Time-Delay Difference Estimation Method for Signal Enhancement in the Distorted towed Hydrophone Array. Remote Sens. 2021, 13, 4586. [CrossRef]

25. Wu, Q.; Zhang, H.; Lai, Z.; Xu, Y.; Yao, S.; Tao, J. An Enhanced Data-Driven Array Shape Estimation Method Using Passive Underwater Acoustic Data. Remote Sens. 2021, 13, 1773. [CrossRef] 
26. Wales, S.C.; Heitmeyer, R.M. An ensemble source spectra model for merchant ship-radiated noise. J. Acoust. Soc. Am. 2002, 111, 1211-1231. [CrossRef] [PubMed]

27. Kay, S. Fundamentals of Statistical Signal Processing, Volume III: Practical Algorithm Development; PTR Prentice Hall: Englewood Cliffs, NJ, USA, 1993. 